\title{
Hausarbeit als theoretische Kategorie eines Emanzipationskonzeptes von Frauen?
}

\begin{abstract}
,Innerhalb der Frauenbewegung lehnen wir ab, daß der Klassenkampf dem Frauenkampf untergeordnet sei, wie auch, daß der Frauenkampf dem Klassenkampf untergeordnet sei. Klassenkampf und Feminismus bedeuten für uns ein und dasselbe."

Erklänung des internationalen Feministinnen-Kollektivs (1)
\end{abstract}

\section{Einleitung}

Ausgelöst wurde die Diskussion um eine gesellschaftliche Anerkennung von Hausarbeit in der Bundesrepublik und Westberlin 1973 mit dem Erscheinen der deutschen Übersetzung der Arbeit Mariarosa Dalla Costas, Die Frauen und der Umsturz der Gesellschaft (2). Die Arbeit erregte erhebliches Aufsehen, basierte die Forderung Dalla Costas nach Anerkennung der Hausarbeit als gesellschaftlich notwendiger Arbeit auf der analytischen lnanspruchnahme einer zentralen Kategorie der marxistischen Analyse, der produktiven Arbeit.

Hausarbeit, Produktion und Reproduktion von Arbeitskraft in physischer, psychischer und sexueller Hinsicht, ist der Ursprung jeder Produktion und jeden Profits und muß aufgrund ihres zentralen Stellenwerts für das kapitalistische Produktionsverhältnis als zentrale Kategorie einer Emanzipationskonzeption für Frauen angesehen wierden.

Auf Basis dieser Analyse formierten sich nach dem Vorbild der in Italien und den angelsächsischen Ländern bereits älteren Diskussion auch in der Bundesrepublik und Westberlin Gruppen, die - hierin allerdings über Dalla Costa hinausgehend (3) eine Bezahlung der Hausarbeit als politische Forderung aufstellten (4).

1 In: Frauen in der Offensive, Lohn für die Hausarbeit oder: auch Berufstätigkeit macht nicht frei, München 1974, S. 13

2 Dalla Costa, Mariarosa, Die Frauen und der Umsturz der Gesellschaft, in: Dalla Costa, Mariarosa/James, Selma, Die Macht der Frauen und der Umsturz der Gesellschaft, Berlin (West) 1973

3 Dalla Costa fordert keine Lohnzahlung, sondern empfiehlt Verweigerung der Hausarbeit bzw. Zerstörung der Hausfrauenrolle, eine auf dem Hintergrund der im wesentlich geringerem Maße berufstätigen und damit finanziell abhängigen italienischen Hausfrau nicht gerade realistische Konsequenz. Über die der Lohnforderung wird noch zu diskutieren sein.

4 Es muß darauf hingewiesen werden, daß die Lohnforderung immer die des Lohns für die Hausarbeit, nicht die des Lohns für die Hausfrau beinhaltet. Männer sind hier also in gleichem Maße angesprochen.

Literatur zum Thema „Lohn für die Hausarbeit":

- Frauen in der Offensive, Lohn für die Hausarbeit oder: auch Berufstätigkeit macht nicht frei, a.a.O.

- Federici, Nicole/Cox, Silvia, Counter-planning from the kitchen, London 1976 
Lohn für Hausarbeit soll nicht nur dazu dienen, die ,Mystifikation der Hausarbeit" aufzubrechen, d.h. Hausarbeit nicht länger als Ausdruck der weiblichen Natur erscheinen zu lassen, er soll der Frau auch die ökonomische Unabhängigkeit verschaffen, um auf dieser Basis, im Rahmen eines antikapitalistischen Kampfes, die geschlechtsspezifische Arbeitsteilung aufheben zu können. Dies beinhaltet gleichzeitig eine Aufhebung jeglicher Form weiblicher Diskriminierung, einschließlich frauenspezifischer Arbeitsplätze, Qualifikationsdefiziten und Lohndifferenzierungen (5).

Lohnzahlung, als gesellschaftliche Form der Anerkennung von Arbeit, soll aber kein Mittel der Integration in das System (6) bilden, sondern die Basis eines gemeinsamen Kampfes (7) für den Aufbau einer Gesellschaft, die durch Integration aller ihrer Mitglieder (8) gekennzeichnet ist und durch Teilhabe aller am gesellschaftlichen Reichtum gleichberechtigte menschliche Beziehungen (9) überhaupt erst ermöglicht.

- Priss, nichtgehaltene Rede in Bremen (i.d. $\dot{U}$. Ute Brockhaus), unveröffentlichtes Manuskript (o.J.)

- Warrior, Betsy, Housework: Slavery or Labor of Love, in: A Journal of Female Liberation - The First Revolution by Cell 16, Cambridge (Massachusetts), Auszug dieses Artikels erschienen in: Notes from the Third Year, S. $212 \mathrm{ff}$.

- Bock, Gisela, Frauenbewegung und die Perspektive „Lohn für Hausarbeit", unveröffentlichtes Manuskript 1976

- dies., Lohn für Hausarbeit und die Macht der Frauen, in: Courage 1/76

- Federici, Sylvia, Wages against housework, London"1975

- Lesbianismus und Macht, Rede auf der internationalen Konferenz der Lohn für Hausarbeitskampagne in London, 1975, unveröffentlichtes Manuskript

- Wages, Housework, Women, Wages for Housework. Women speak out, Toronto 1975

- Warrior, Betsy und Leghorn, Lisa, Houseworker‘s Handbook, Cambridge, Mass., 1974

5 Dem Ansatz der Bewegung ,Lohn für Hausarbeit“" entsprechend wird die häusliche Diskriminierung der Frau als zentrale Ursache für außerhäusliche Diskriminierungsformen gesetzt.

6 Die Vertreterinnen der Lohnforderung befinden sich hier unter starken Legitimationszwängen. Die Einwände einer Institutionalisierung kommen nicht nur von seiten der gegnerischen ,Linken“, sondern auch von Teilen der Frauenbewegung selbst. Vgl. dazu

- Schwarzer, Alice, Der kleine Unterschied und seine großen Folgen, Ffm. 1975

- Krechel, Ursula, Selbsterfahrung und Fremdbestimmung, Darmstadt und Neuwied 1975

- Stefan, Renate, Hausfrauen und Mütter, die vergessenen Sklavinnen, Berlin (West) 1975 Der Institutionalisierungseinwand drängt sich umso mehr auf, als die Vertreterinnen der Lohnforderung sich hier mindestens oberflächlich auf einer Argumentationsebene mit den Erziehungsgeldforderungen bürgerlicher Parteien befinden.

7 Die artikulierte Gemeinsamheit enthält Vorstellungen einer Autonomie des Frauenkampfes im Rahmen der Arbeiterbewegung. Die konkrete Umsetzung dieses vorläufig noch theoretischen Problems wird für die weitere Entwicklung der Frauenbewegung von entscheidender Bedeutung sein. Zur Zeit zeigen sich noch erhebliche Widerstände von beiden Seiten. Zum Problem der Autonomie vgl. auch

- Krechel, Ursula, a.a.O.

- Schuhler-Klitzing, Juliane, Women's Liberation: die amerikanischen Frauen kämpfen für ihre Befreiung, in: Mitchell, J., u.a., Frauenemanzipation, München 1974

8 Gemeint sind u.a. Kranke, Alte, Kinder.

9 Auch Beziehungen zwischen den Geschlechtern. 
Nur im Kampf für điese Gesellschaft kann die Frau die „Kastration“ ihrer „,produktiven Kreativität“", ihrer „sexuellen, psychischen und emotionalen Autonomie" überwinden und eine Identität entwickeln.

Die mit der Forderung nach Bezahlung der Hausarbeit verbundene Hoffnung auf eine Integration in die Arbeiterklasse drückt nicht nur die bisherige Vernachlässigung der Hausfrau für eine gewerkschaftliche Organisierung aus, sie ist gleichzeitig durch die Einsicht geprägt, daß die Aufhebung der geschlechtsspezifischen Arbeitsteilung auch im lnteresse der männlichen Lohnarbeiter liegen muß.

Um die theoretische und politische Brisanz dieser Forderung überhaupt wahrnehmen zu können, bedarf es einer gewissen Bereitschaft, den Denkanstoß durch die in Form und Inhalt provokante Argumentation aufzugreifen.

Eine Provokation bildet diese „Bewegung“ ohne Zweifel. Nicht nur hinsichtlich ihrer wissenschaftstheoretischen Unkonventionalität, die es - wie noch zu zeigen sein wird - zuläßt, zentrale Prämissen ihrer Strategie auf rein deklamatorische Behauptungen zu stützen, sondern auch hinsichtlich ihres Vorwurfs an die Linke (10), sie spalte durch Rassismus und Sexismus (11) die Arbeiterklasse und sei im antikapitalistischen Charakter ihrer Strategie(n) nicht ernst zu nehmen.

Was in beschriebener Weise formuliert, trifft nicht nur den wunden Punkt traditionell sozialistischer Theorie der Emanzipation der Frau, die die ökonomische Unabhängigkeit der Frau nur über den Weg einer außerhäuslichen - gesellschaftlichen - Arbeit erlaubt, damit aber dem Problem der Doppelbelastung und seiner Auswirkung auf die erhoffte emanzipatorische Wirkung von Arbeit relativ hilflos gegenübersteht, son dern auch den Kern der Emanzipationsdebatte überhaupt, nämlich die Frage des analytischen Primats von Patriarchat oder Kapitalismus.

In den Anfängen der Frauenbewegung (12) in der Bundesrepublik und West-

10 „Linke“ umfaßt alle die, die in der Ablehnung der Arbeit im Haus als bedeutungslos für die Reproduktion des Kapitals die Bedeutungslosigkeit der Hausfrau für gesellschaftsbeeinflussende Strategien ausdrücken und ihr die Möglichkeit einer befreienden Veränderung nur über den Weg von Zusatzarbeit zusprechen.

11 Weil sie das „Problem von Machtstrukturen innerhalb der ausgebeuteten Klasse" ignoriert und den spezifissch unterdrückten Gruppen eigenständige Forderungen abspricht. Vgl. dazu

- Erler, Gisela, Die Rolle der Frau und Machtstrukturen, in: Frauen in der Offensive, a.a.O.

- James, Selma, Zum Verhältnis von Geschlecht, Rasse und Klasse, in: Frauen in der Offensive, a.a.O.

12 Als ,Neue" oder ,Zweite" Frauenbewegung wird der Prozeß der Bildung neuer Arten von autonomen Frauengruppen in den meisten europäischen und nordamerikanischen Industriestaaten seit ungefähr 1968, in den letzteren etwas früher, bezeichnet. Die historische Vorläuferin dieser Bewegung, die ..Alte" oder „Erste" machte ihre ersten Gehversuche bereits im Gefolge der Frz. Revolution, konstituierte sich allerdings erst um die Mitte des 19. Jhdts., zunächst in den USA, dann in England und dem übrigen Europa. Um unterschiedliche Organisations-, Praxis- und Zielvorstellungen aufzuzeigen, wird sie allgemein in ,bürgerliche" und ,proletarische" Frauenbewegung unterteilt. Die Literatur zur „Alten“" Frauenbewegung ist zahlreich. Es finden sich meist kurze Zusammenfassungen in den neueren Standardwerken der Frauenliteratur, so

- Beauvoir, Simone de, Das andere Geschlecht, Reinbek b. Hamburg, 1968 
berlin zentrierten sich um diese methodischen Fragestellungen noch sich heftig befehdende Gruppierungen, die schlagwortartig mit ,Feministinnen“, von liberal bis radikal, und „Sozialistinnen " bezeichnet wurden.

Machten die „Sozialistinnen“" private Aneignung gesellschaftlicher Arbeit die spezifische Produktions- und Herrschaftsform des Kapitalismus - als Ursache für die Unterdruickung der Frau verantwortlich und sahen deren Aufhebung und die Konstituierung einer sozialistischen Gesellschaftsordnung als Voraussetzung für eine freie Entfaltung der Frau an, herrschte bei den „Feministinnen“ Einigkeit darüber, daß die Ursache der Unter drückung der Frau nur in der geschlechtsspezifischen Herrschaft des Mannes, dem Patriarchat, zu finden sei. Eine herrschaftsfreie Gesellschaft war nur durch die Aufhebung aller geschlechtsspezifischen Unterschiede über den Weg einer „Geschlechterrevolution "möglich (13), die Konzeptionen von Aufhebung der traditionellen Geschlechterrollen bis hin zur Abschaffung des Geschlechtsunterschiedes (14) umfaßte.

Klassenkampf oder Geschlechterkampf, so stellte sich die strategische Alternative. War dèn einen die linke Analyse nicht radikal genug (15), wurde bei den anderen Feminismus mit unpolitisch und antisozialistisch gleichgesetzt (16).

Man wird dieser Problematik nicht gerecht, reflektiert man sie nicht als Erscheinungsform eines komplexen Zusammenhangs im historischen Kontext von Entstehung und Entwicklung der ,Neuen Frauenbewegung“"

- Menschik, Jutta, Gleichberechtigung oder Emanzipation, Ffm 1971

Literatur zur Neueren Frauenbewegung

- Cipa, Angelika u.a., Zur Entwicklung der Frauenbewegung in den USA, Frankreich und der BRD in: Blätter für deutsche und internationale Politik 7/74

- Linnhoff, Ursula, Die Neue Frauenbewegung, Raubdruck

- Nave-Herz, Rosemarie u.a., Die Ziele der Frauenbewegung, in: Beilage zur Wochenzeitung ,Das Parlament", 13. Dez. 1975

Entsprechend der Schwierigkeit, die unzähligen Frauengruppen erfassen zu können, muß die Literatur noch als analytisch unzureichend bezeichnet werden. Die Ubergänge der gekennzeichneten Einteilungen in der Einleitung dieser Arbeit sind selbstverständlich fließend.

$13 \mathrm{Zu}$ beachten ist eine Operationalisierung des Begriffs der Revolution. Seine auch für Feministinnen typische Herauslösung aus dem sozialhistorischen Zusammerhang hat zur Folge, daß, da , sie nicht in der Lage sind, und im Namen ihrer reinen und totalen Revolution handeln, (. . .) sie zumeist empört über die Flexibilität und Integrationsfähigkeit des bekämpften, Systems (sind)" vgl. Mühlbauer, Rita, Strategiemodelle der Neuen Frauenbewegung, in: Albus, Anita u.a., Maskulin-Feminin, München 1975. Da es sich'bei diesem bereits 1972 geschriebenen Aufsatz nach Kenntnis der Verfasserin um die beste bislang existierende Analyse der Frauenbewegung handelt, bezieht sich die Einleitung in wesentlichen Teilen auf diese Arbeit.

14 Allerdings nicht im Sinne einer Valerie Solanas, die 1969 erhebliches Aufsehen erregte mit ihrem Buch "Manifest der Gesellschaft zur Vernichtung der Männer (SCUM)", sondern in dem der von Firestone, Shulamith, Frauenbefreiung und sexuelle Revolution, Ffm 1975, am präzisesten konzipierten Formation eines kybernetischen Sozialismus, in dem die Wahl künstlicher Reproduktion die Frau von ihrem Gebärzwang befreien könnte. Nach neueren Nachrichten aus England ja wohl medizinisch möglich.

15 vgl. Firestone, Shulamith, a.a.O., S. 40

16 vgl. Haug, Frigga, Verteidigung der Frauenbewegung gegen den Feminismus, in: ,Das Argument" 83, Dez. 1973, S. 938 
Die „Neue Frauenbewegung“ ist aus der antiautoritären Bewegung hervorgegangen. Inwieweit die Entstehungsbedingungen der antiautoritären Bewegung auch zur Analyse der Frauenbewegung herangezogen werden können, ist im einzelnen noch nicht untersucht worden. Man kann jedoch davon ausgehen, daß das Infragestellen aller bisherigen Werte und Normen des Etablierten durch die antiautoritäre Bewegung - sowohl ihre kulturelle als auch moralische Dimension - auch den Frauen einen Ansatz zur Revolte bot. Die theoretischen und praktischen Mittel der Neuen Linken wurden zur Analyse der eigenen Unterprivilegiertheit benutzt und als Reaktion auf das Unverständnis der männlichen Linken gegen diese selbst eingesetzt.

Die frühe spontaneistische Phase der Frauen bewegung war nicht von einer legitimierten Emanzipationstheorie getragen. „Eine solche gab es nicht. Erst mittels der Erfahrungen in der Aktion sollte eine Theorie entwickelt werden. Das proklamierte Aktionsziel war die Erotisierung aller Lebensbereiche, d.h. die Negation des männlichen Leistungsprinzips. ${ }^{66}(17)$

Für die Erarbeitung einer solchen Theorie spielte zweifelsohne eine entscheidende Rolle, daß die Frauenbewegung zu einem Zeitpunkt entstand, an dem die antiautoritäre Bewegung sich ihrem Ende zuneigte und in eine Dogmatisierungsphase trat, die sich in der Bundesrepublik und Westberlin besonders stark auswirkte. „Die feministischen Auftritte paßten nicht ins Konzept einer Bewegung, die sich immer mehr auf ein angeblich proletarisches Erbe besann und zu diesem Zeitpunkt alle Anstrengungen unternahm, ihren en ttäuschten Idealismus und ihre Hoffnungen auf Identität in die Geschichte der Klassenkämpfe hineinzuretten."(18) Der damit notwendig gewordenen politischen Legitimation konnten sich besonders die Frauen nicht entziehen, die sich aus politischen Organisationen rekrutierten (19).

Inwieweit man daraus schließen kann, daß es zuerst die politischen Frauen waren, die die Spaltung in einen antikapitalistischen un d einen antipatriarchalischen Kampf vollbrachten, indem sie mit ihrer Betonung des allgemeinen Kampfes dem Frauenkampf eine untergeordnete Rolle zuordneten (20), ist wahrscheinlich weniger wichtig zu diskutieren als die Frage, was sich hierin ausdrückt. Die radikale Infragestellung gesellschaf tlicher Identifikationsmuster weiblicher Subjektivität muß als historisch beispiellos angesehen wer den. Dem Verlust des eigenen Standorts entsprach die Suche nach einer Identität. Die Erfahrungsebene der Konfrontation hatte die Sensibilisierung für Unterdrückungsformen verschärft. Die Ablehnung aller Maßstäbe als männlich formulierter warf auf das eigene Geschlecht zurück. Kollektive Selbsterfahrung wurde zum Mittel der Aneignung eigener und gesellschaftlicher Realität. Der theoretischen und praktischen Suche nach der historisch, verschütteten" Frau diente dem Konstitutionsprozeß einer neuen Gesellschaft, die als solche Befreiung für alle versprach (21).

\footnotetext{
17 Mühlbauer, Rita, a.a.O., S.224 f。

18 ebenda S.228

19 In Westbérlin läßt sich diese Entwicklung anhand des ,Sozialistischen Frauenbundes“ nachvollziehen.

2o vgl. „,Schwarze Protokolle", Januar 1974, S.12

21 Der theoretischen Position entspricht das Beispiel der in den USA entwickelten Women's
} 
Daß dieser Konstitutionsprozeß nur bruchstückhaft in eine Theorie bil dung einging, muß wesentlich darin gesehen werden, daß die im Patriarchalismusvorwurf liegende Totalität nahezu die gesamte Menschheitsgeschichte als ein erneutes Untersuchungsobjekt erfor derlich machte.

Den der politischen Legitimation unterliegenden Frauen erlaubte sich diese maßstabsetzende Zentrierung auf das eigene Geschlecht nicht. Nicht Negation (der von Männern geschaffenen) gesellschaftlichen Bedingungen, son dern umgekehrt, die Wahrnehmung und Analyse derselben unter dem Aspekt ihrer Unterdrückungsmomente für beide Geschlechter, war das Erge bnis ihres Rückgriffs auf die Klassiker der sozialistischen Theorie. Denn erst im Prozeß der aktiven Verän derung dieser Unterdrückungsmomente entwickeln die Frauen ihre Fähigkeiten und die zukünftige Basis gleicher Ausgangsbe dingungen für beide Geschlechter.

Da die Frau aufgrund ihrer spezifischen Benachteiligung durch Mutterschaft, Ehe und Haus eine Einschränkung ihrer gesellschaftlichen Teilnahme erfährt, gilt es diese geson dert zu fördern. Aufgrund mangelnder Berücksichtigung des historischen Gehalts der von ihnen adaptierten Theorie erforderte ihre Identitätssuche im Prozeß der Veränderung der Gesellschaft ein hohes Maß an Selbstverleugnung, wurde die Wahrnehmung weiblicher Diskriminierungsformen - die Gemeinsamkeit des Klassenkampfs nicht gefährdend - nur in ihrer systembeding ten Abstraktion erlaubt. Ein wesentlicher Bereich der konkreten täglichen Erfahrungsebene blieb so notwendigerweise ausgeklammert.

Der Ausschließlichkeitsanspruch, der beiden Vorgehensweisen innewohnte, prägte die die Frauenbewegung konstituierenden Widersprïche von sozioökonomischen Gegensätzen und Frauensolidarität, von Selbstverständnis und Praxis und kennzeichnet die bereits genannte dominierend theoretische Auseinandersetzung über die Vorrangigkeit von Patriarchat oder Kapitalismus. Die Frauenbewegung auf ihrem Entwicklungshintergrund zu reflektieren, heißt, die skizzierten Probleme auch ausge drückt in ihren Theorien zu bedenken und heißt weiterhin, die alternativen Strategien des Geschlechter- oder Klassenkampfs als noch unausgereiften Stand einer jungen Bewegung zu akzeptieren.

Auf diesem Stand ist die Frauenbewegung allerdings weder theoretisch noch strategisch in der Eage, der Komplexität der Emanzipationsproblematik gerecht zu werden (22). Dies erweist sich als entscheidendes Hindernis ihrer Mobilisierungswirkung.

Die Ausweitung des Forschungsstandes und die Vervielfältigung der Erfahrungsebene hat - so eine These der Verfasserin - die anfänglich politische Konfrontation zugunsten einer Vielzahl theoretischer und praktischer Projekte in den Hintergrund treten lassen und wird - in Ansätzen bereits sichtbar - den Alternativcharakter von Kapitalismus und Patriarchat zugunsten einer ihre Verbindung reflektie-

Studies, ein interdisziplinärer Wissenschaftsbereich, in dessen Zentrum die Erforschung der Frau in der patriarchalischen Gesellschaft steht, der praktischen Beispiele wie Frauenverlage, Frauenzeitungen, -druckereien, -buchläden, -banken, etc.

22 vgl. dazu auch Prokop, Ulrike, Weiblicher Lebenszusammenhang. Von der Beschränktheit der Strategien und der Unangemessenheit der Wünsche, Ffm 1976 
renden Analyse reduzieren. Die Effektivität der Frauen bewegung wird weitestgehend davon abhängen, inwieweit auf Basis solcher Analysen die Strategien zu vervielfältigen sind und ihre Kombination politisch möglich sein wird.

Auf diesem Hintergrund erweist sich die Relevanz der Forderung nach Anerkennung der Hausarbeit.

Sie greift den Tatbestand auf, daß weder kapitalistische noch sozialistische Gesellschaften auf die private Wahrnehmung familialer Reproduktionsarbeit - besonders im Falle von Kindern - verzichten können, daß diese Arbeiten nahezu ausschließlich von Frauen ausgeführt werden und daß in kapitalistischen Gesellschaften die von Sozialistinnen erhoffte emanzipatorische Wirkung außerhäuslicher Arbeit ihre Einschränkung nicht nur durch die Auswirkung der Doppelbelastung auf die Frauen selbst erfährt, sondern der ,geschlechtsspezifische Arbeitsmarkt ${ }^{\text {‘ }}$ sich ja gerade dadurch auszeichnet, daß er die Doppelbelastung als profitables Moment in seiner Struktur aufgegriffen hat.

Der Versuch, auf Basis dieser Erkenntnisse die Hausarbeit analytisch als Arbeit zu erfassen, dient nun nicht dem Zweck, diese als ,,vorkapitalistische Arbeit" in ihrer Auswirkung auf das, ,rückständige politische Bewußtsein" der (Haus)Frauen zu analysieren und daraus die Legitimation für die Notwendigkeit eines (zusätzlichen) außerhäuslichen Arbeitsplatzes abzulenken, sondern dazu, den Widerspruch von gesellschaftlich notwendiger Arbeit und ihrer gesellschaftlichen Nichtbewertung aufzuzeigen, diesen Widerspruch in seiner Auswirkung auf die betroffenen Frauen zu analysieren und nach seinen systemstabilisierenden Ursachen zu fragen. Der antikapitalistische Charakter der strategischen Konsequenz wird nicht in einer Ablehnung, sondern im Gegenteil in einer Anerkennung der Hausarbeit durch die dieser Gesellschaft entsprechenden Form, das Geld, gesehen. Geld, als sichtbarer Ausdruck von Arbeit, kann entsprechend der Analyse nicht bedeuten, auch die Arbeit qualitativ zu akzeptieren, denn eine Distanzierung von der Arbeit setzt ja deren Wahrnehmung als Arbeit überhaupt erst voraus; das Geld stellt gleichzeitig die materiellen Möglichkeiten, die Ablehnung der Arbeit und mit ihr verbundener Abhängigkeitsverhältnisse auch praktizieren zu können.

Der Ansatz ist insofern für die Emanzipationsdiskussion nicht nur in seiner Kritik an der Sozialistischen Emanzipationstheorie interessant, als er die Frage nach einer theoretischen und strategischen Vernachlässigung des familialen Reproduk tionsbereiches stellt, er ist vor allem deshalb interessant, weil er die Aufhebung der geschlechtsspezifischen Arbeitsteilung in einen gesellschaftsanalytischen Zusammenhang stellt und damit die zentralen Forderungen beider bislang alternativ diskutierten Strategien miteinan der verbindet.

Inwieweit die Kategorie der Hausarbeit theoretisch ein Verbindungselement kapitalistischer und patriarchalischer Strukturen darstellt, kann in der Arbeit nicht geklärt werden, wäre jedoch weiterführend zu überdenken.

Es muß bereits hier - weil aus Gründen des Umfangs in diesem Rahmen nicht darstellbar (23) - festgestellt werden, daß den Vertreterinnen der Forderung nach

23 Es handelt sich bei dieser Arbeit um die Zusammenfassung einer Diplomarbeit gleichen Titels, die 1977 am Otto-Suhr-Institut der FU Berlin (West) geschrieben wurde. Die Dar- 
Anerkennung der Hausarbeit die zentrale Beweisführung der Hausarbeit als kapitalistisch notwen diger Arbeit nicht gelingt.

Dies hat im wesentlichen methodische Grün de, ändert nach Meinung der Verfasserin aber nichts an der Relevanz dieser Fragestellung für den skizzierten Zusammenhang.

Aus diesem Grund soll die Fragestellung im folgenden aufgegriffen und weitergeführt werden, um anhand ihrer Einschätzung den Stellenwert einer Kategoriẹ der Hausarbeit für ein Emanzipationskonzept bestimmen zu können.

Soll te die Hausarbeit ihren - im Sinne einer kapitalistischen Formbestimmtheit - gesellschaftlich notwendigen Charakter erweisen, steht ihr - soweit ist den Vertreterinnen der Lohnforderung zuzustimmen - auch die dieser Gesellschaft entsprechende Form der Anerkennung, das Geld, zu.

Die Berechtigung dieser Forderung unterstellt, erweist sich die Frage nach der systembedingten Funktionalität ihrer bisherigen Nichtbewertung als relevant, um den antikapitalistischen Anspruch der Strategie klären zu können.

Für den Vêrlauf der Arbeit seien noch folgende Voraussetzungen angemerkt: auf die Entstehungsbedingungen der geschlechtsspezifischen Arbeitsteilung kann nicht eingegangen werden, d.h. die geschlechtsspezifische Festlegung der Frau auf die Tätigkeit des privaten Reproduktionsbereiches wird vorausgesetzt, die Frau als Hausfrau wird idealtypisch verwandt, d.h. als solche wird die Frau immer als NichtLohnarbeiterin und somit abhängig vom Mann definiert. In dieser Vorgehensweise ist von der Situation abstrahiert, daß Zuweisung und Ausführung familialer Arbeit im Zusammenhang mit einer zusätzlichen außerhäuslichen Arbeit geschehen.

\section{Der gesellschaftliche Stellenwert von Hausarbeit}

\subsection{Kritik an der „Konzeption" der produktiven Arbeit}

Der Schwerpunkt der Diskussion um die Anerkennung von Hausarbeit liegt auf der Institutionalisierung der Hausarbeit, dem Realitätsgehalt einer Forderung nach Lohn für die Hausarbeit und dem „Konzept" der produktiven Arbeit. Wird die 1nstitutionalisierung der Hausarbeit zusammen mit dem Realitätsgehalt der Lohnforderung abschließend aufzugreifen sein, soll im folgenden die Diskussion um die Hausarbeit als mehrwertschaffende Arbeit nachvollzogen werden.

Die Vertreterinnen der Forderung nach Anerkennung der Hausarbeit bleiben - wie eingangs erwähnt - den Nachweis ihrer zentralen These mit Hinweis auf noch folgende Arbeiten schuldig. Die Kritik an der „Konzeption“ der produktiven Hausarbeit konzentriert sich im wesentlichen auf zwei Punkte (1):

stellung und Analyse der „Bewegung Lohn für Hausarbeit“ fielen in diesem Rahmen dem Umfang zum Opfer. Die Ergebnisse müssen deshalb in Kurzform eingebracht werden.

Die Darstellung bezieht sich ausschließlich auf

- Dalla Costa/James and the Subversion of Marxism: A Critique, in: Women and Revolution No. 5, 1974, und

- Gerstein, Ira, Domestic Work and Capitalism in: Radical America, vol.7, nos. 4/5 July- 
1. den Unterschied zwischen industrieller und privater Konsumtion (i.e. familialer Konsumtion)

2. den zwischen produktiver Arbeit als mehrwertschaffender und einfacher als gebrauchswertschaffender Arbeit.

Der erste Punkt greift einen Aspekt der spezifisch kapitalistischen Form notwendiger Einheit von Produktion und Konsumtion auf, nämlich den, daß die private als individuelle Konsumtion in einem von dem der industriellen Konsumtion unterschiedenen Bereich - der Familie - stattfindet.

Die Kosten der individuellen Konsumtion stellen zwar --als Lohnkosten -- einen Faktor der in dustriellen Produktion dar, die individuelle Reproduktion selber ist aber keine kapitalistische Produktion. Da die Kategorie der produktiven Arbeit sich auf den Verwertungsprozeß des Kapitals bezieht, erfordert die Annahme der Hausarbeit als produktiver die Annahme von Kapitalverhältnissen für die Familie. Dies hieße, entweder die Arbeiterklasse als Produzenten und Besitzer der Arbeitskraft zum Besitzer des von ihnen geschaffenen Mehrwerts zu erklären (2) oder aber den Kapitalisten zum Besitzer der Familie (3).

Beides, nicht ohne Phantasie, bedarf je doch wohl kaum der ernsthaften Diskussion. Sowenig der Arbeiter Besitzer des Mehrwerts ist, sowenig besitzt der Kapitalist den Arbeiter, denn gerade durch letzteres, nämlich die „Freiheit" des Sich-verkaufen-Müssens, unterscheidet sich der Kapitalismus von vorkapitalistischen Gesellschaftsformationen.

Es kann so weder die Produktion und Aufzucht von Nachkommen als kapitalistische Warenproduktion gefaßt werden, denn die im Menschen angelegte und reproduzierte potentielle Arbeitsfähigkeit realisiert erst auf dem Markt ihren Warencharakter, noch die Reproduktion der Ware Arbeitskraft, denn diese - so die inhaltliche Aussage des zweiten Punktes - ist nicht kapitalistische, sondern einfache Warenproduktion. Sie erfolgt nicht unter dem Gesichtspunkt der Verwertung des Werts, rastloser Motor kapitalistischer Warenproduktion, sondern unter dem der Konsumtion (4). lst die Verwertung des Werts aus der Sicht des Einzelkapitals unbegrenzt, so ist es die Konsumtionsfähigkeit des Individuums nicht. Sie findet ihre Grenze in seiner historisch und moralisch definierten Aufnahmekapazität. Die Haus'arbeit ist gebrauchswertschaffende Arbeit (5). Der Wert der Ware Arbeitskraft setzt sich zusammen aus dem der konsumierten Lebensmittel (im weitesten Sinne) und dem der zu seiner Aufrechterhaltung nötigen Dienste. Die Hausarbeit ist hier als wertübertragende oder werterhalten de Arbeit nicht berïcksichtigt. Dies heißt nicht, daß Frauen im Haus nicht arbeiten, aber Hausarbeit ist keine kapitalistische Arbeit

October 1973. Beide Vertreter(innen) können als typisch für diesen Argumentationsverlauf angesehen werden.

2 vgl. Gerstein, Ira, a.a.O., S.114

3 vgl. Dalla Costa/James and the Subversion of Marxism ..., a.a.O., S.4

4 vgl. Gerstein, Ira, a.a.O., S.113

5 Zum Aspekt der Hausarbeit als einfacher Gebrauchswertproduktion vgl. auch Benston, Margaret, The Political Economy of Women's Liberation, in: Monthly Review, vol.21, No. 4 (Sept. 1969). Übersetzung dieses Artikels in: Mitchell, J. u.a., Frauenemanzipation, München 1974 
(6). Da die Hausarbeit nur Gebrauchswerte, keine Werte schafft, ist die Forderung nach Bezahlung der Hausarbeit unberechtigt. Hausarbeit und Lohnarbeit sind voneinander unabhängige ökonomische Kategorien, die - auf unterschiedlichen sozialen Beziehungen beruhend - ihren eigenen Gesetzen gehorchen. Eine wesentliche Mystifikation dieser Gesellschaft besteht allerdings daran, daß Hausarbeit als ökonomische Kategorie identifiziert wird mit weiblicher Arbeit, ein Aspekt, der auf die geschlech tsspezifische Arbeitsteilung zurückzuführen ist. Nicht weil Frauen diese Arbeit machen, schafft Hausarbeit keine Werte, sondern aus ihrem Charakter als Hausarbeit heraus (7).

\subsection{Die gesellschaftliche Notwendigkeit von Hausarbeit}

Die Hausfrau arbeitet also, diese Arbeit ist aber keine wert-, schon gar nicht mehrwertschaffende Arbeit, so das Fazit des letzten Abschnittes, dem erst einmal so weit gefolgt werden kann, als die Hausarbeit keine mehrwertschaffende Arbeit ist.

Der dargestellte Argumentationsgang ist Ausdruck einer Gesellschaftsanalyse, die die Konstituierung kapitalistischer Form von Gesellschaft folgendermaßen faßt. Die Mitglieder dieser Gesellschaft stellen ihren gesellschaftlichen Zusammenhang nicht mehr unmittelbar - für sie wahrnehmbar - im Akt gemeinschaftlicher Produktion/Reproduktion her, sondern nur noch mittelbar über den Austausch, zudem verfremdet in der Form von Waren, respektive ihrem Wertausdruck in Geld.

Dem vorausgesetzt und impliziert ist der Warencharakter von Arbeit. Das bedeutet, daß als Resultat von Arbeitsteilung und spezifischer Besitzverhältnisse dem Menschen das fremd - oder entfremdet - wird, was als „Wesenstätigkeit" seine spezifisch menschliche Fähigkeit ausmacht, sich selbst und seine Umwelt schaffen, erfassen und verändern zu können, die Arbeit. Arbeit, eigentlich Zweck der Befriedigung von Bedürfnissen, wird so zu einem bloßen Mittel, das wahrgenommen werden muß, um Bedürfnisse, nun außerhalb dieses Bereichs liegend, realisieren zu können. Entfremdung umfaßt so den Inhalt der Arbeit, das Produkt dieser Arbeit, Entfremdung von sich selbst und seinem gesellschaftlichen Zusammenhang, wird aber gleichzeitig definiert als Übergangsstadium, das durchlaufen werden muß, um die objektiven und subjektiven Bedingungen seiner Aufhebung hervorbringen zu können.

Da der Hausarbeit Warenform abgesprochen wird, fällt die hier geleistete Arbeit aus dem beschriebenen Zusammenhang heraus, mit dem bemerkenswerten Ergebnis, daß Arbeit, die der - jeder Gesellschaft unentbehrlichen - Produktion und Reproduktion der Gattung dient, entweder auf die Stufe privater Arbeit, die ihren gesellschaftlichen Charakter nicht ausdrïcken kann, verwiesen wird, damit je nach

6 Dalla Costa/James and the Subversion of Marxism ..., a.a.O., S.4

7 Gerstein, Ira, a.a.O., S.119.

Dient den Autoren. in "Women and Revolution" das Untersuchungsergebnis ausschließlich dazu, die Notwendigkeit der außerhäuslichen Arbeit zu bekräftigen, ist Gersteins Aufsatz - im Gegensatz dazu - durch ein wesentlich differenzierteres Aufgreifen dieser Problematik gekennzeichnet. 
Gesichtspunkt verschwendete Arbeit oder schlicht Privatvergnügen ist, oder aber als vorkapitalistische Arbeit in der historischen Auflösung begriffenes Relikt ist. Für das Subjekt dieser Arbeit, die Hausfrau, bedeutet dies den Ausschluß aus einer wenn auch entäußerten Form ihrer Gesellschaftlichkeit, die Konstituierung ihrer eigenen individuellen und gesellschaftlichen Existenz nicht ,an ihr ${ }^{66}$, sondern schlichtweg ohne sie, und eine tätige Konstituierung ihrer individuellen und gesellschaftlichen Existenz nur um den Preis doppelter Arbeit.

In dieser Form muß die Analyse sich geschlechtsspezifische Diskriminierung vorwerfen lassen.

Um die Hausarbeit dennoch als gesellschaftlich notwendige Arbeit begreifbar zu machen, erweist es sich als notwendig, einige Aspekte der gesellschaftlichen Formbestimmtheit des Geldes hinzuzuziehen.

Um sich zu reproduzieren, verkauft der Lohnarbeiter seine Arbeitskraft. Mit der Lohnform ist die spezifische Eigentumsform des Lohnarbeiters gegeben. Sie macht ihn zum Privateigentümer, als solchen gleich allen anderen Privateigentümern. Verschwunden ist in dieser Form die Notwendigkeit des an Produktionsmitteln besitzlosen Arbeiters, seine Arbeitskraft àls Ware auf dem Markt verkaufen zu müssen und erscheint als freier Willensakt seiner selbst. Der Lohn stellt weiter die spezifische Form der Teilhabe des Arbeiters am gesellschaftlichen Reichtum dar und setzt als familiale Beitragsform des Mannes in seiner Quantität den Rahmen privat zu betreibender Ökonomie. ,Der Selbständigkeit der Eigentümer auf dem Markt entspricht eine Selbstdarstellung der Menschen in der Familie. Deren, wie es scheint, vom gesellschaftlichen Zwang gelöste Intimität ist das Siegel auf die Wahrheit einer im Wettbewerb geübten Privatautonomie. Private Autonomie, die ihren ökonomischen Ursprung verleugnet, eine außerhalb des Bereichs der durch den autonom sich dünkenden Marktteilnehmer einzig praktizierten, verleiht denn auch der bürgerlichen Familie das Bewußtsein ihrer selbst. Sie scheint freiwillig und von freien Einzelnen begründet und ohne $Z$ wang aufrechterhalten zu werden. "(8)

Für die Frau stellt es sich nun so dar, daß sie als Hausfrau nicht über ihre Arbeitskraft als Ware verfügt. Um sich zu reproduzieren, verkauft sie demnach ihre Arbeitskraft nicht, sondern sie heiratet. Die Ehe erscheint aber nicht als ein Mittel zur Reproduktion (9), sondern letztere wird untergeordnetes Element eines übergeordneten Zusammenhangs, der Familie. Definitionsgemäß erlaubt diese unter Berufung

8 Habermas, Jürgen, Strukturwandel der Öffentlichkeit, Neuwied und Berlin 1971 (5.Aufl.) S.64. Diese bürgerliche Fiktion der freien Verfügbarkeit über eigenen Besitz wird im übrigen darin unterstützt, daß das private „Haushalten“ den Anschein hat, daß die Selbsterhaltung in eigener Regie organisiert wird. Vgl. dazu Milhoffer, Petra, Familie und Klasse, Ffm 1973, S.69

9 Man kann davon ausgehen, daß die gesellschaftlichen Alternativen, die Frauen zugestanden werden, nach wie vor ausschlaggebend dafür sind, inwieweit die Ehe als Mittel der Reproduktion wahrgenommen werden muß. Ist die ,Liebesheirat" historisch gesehen ohnehin neueren Datums, entbehrt diese Bezeichnung erst dann ihres ideologischen Charakters, wenn sie nicht mehr die Ausnutzung emotionaler Bedürfnisse für gesellschaftlich unbezahlte Reproduktions- und Aufzuchtsarbeit - zudem noch auf Kosten eines ganzen Geschlechts - beinhaltet. 
auf die natürliche und sittliche Ordnung rechtlich abgesicherte Arbeitsteilung (10) nicht, die familialen Leistungen der Frau als Arbeit, die der eigenen Reproduktion dient, zu betrachten, sondern als Pflichten, die der Aufrechterhaltung der Familie geschuldet sind. Um diese aber wahrnehmen zu können, muß die Reproduktion der Frau gewährleistet sein. Entsprechend bestehen die Pflichten des Mannes gegenüber der Familie in der Versorgungsleistung, „Haushalts- und Berufstätigkeit sind, von der Familie aus gesehen, beide unerläßličc." (11) Die Frau geht so als Nicht-Besitzerin in die Ehe ein (12), d.h. wie dargestellt, sie verkauft ihre Ware Arbeitskraft in der Ehe nicht, erhält dementsprechend auch nicht den Wert ihrer Ware in Geld.

Die damit gesetzte direkte Abhängigkeit von ihrem Ehemann erweist ihre Notwendigkeit als Zwang zur Wahrnehmung gesellschaftlicher Aufgaben, denn die Familie spielt - so Habermas - ihre genau umschriebene Rolle im Verwertungsprozeß des Kapitals, nicht nur hinsichtlich der Schaffung einer personellen Kontinuität, die die Akkumulation des Kapitals gewährleistet, sondern vor allem dient sie, als eine Agentur der Gesellschaft, der Aufgabe jener schwierigen Vermittlung, die beim Schein der Freiheit die strenge Einhaltung der gesellschaftlich notwendigen Forderungen dennoch herstellt (13).

Die Besitzlosigkeit der Frau macht ihre Abhängigkeit von der Beitragsform des Mannes - dem Lohn (14) - aus, denn nur mittels diesem können die zur Repro-

10 Vgl. Simitis, Spiros, Zur Situảtion des Familienrechts - über einige Prämissen, in: Simitis, S./Zenz, G. (Hrsg.), Seminar Familie und Familienrecht, Bd. 1, Ffm 1975, S. 17. Simitis verweist in diesem Zusammenhang auf die gesellschaftliche Relevanz der Familie angesichts der Tatsache, daß die Heraushebung der Familie aus der Ebene einer rational zugänglichen Argumentation ihren Stellenwert in der Durchsetzung, Festschreibung und Immunisierung von Normen zum Zwecke der Verteidigung einer bestimmten Vorstellung von Familie findet, die als solche dann als rechtlich einzig tolerabel hingestellt wird.

Die Normen unterliegen zwar der historischen Veränderung, nicht aber deren Bedeutungsgehalt als Konkretisierung der natürlichen und sittlichen Ordnung.

11 Müller-Freienfels, Wolfram, Gleichberechtigungsprinzip und eheliches Güterrecht, in: Simitis, S./Zenz, G. (Hrsg.), a.a.O., S. 263

12 Die vermögensrechtliche Stellung der Frau war bis zum Inkrafttreten des Gleichberechtigungsgesetzes 1953 dadurch gekennzeichnet, daß - brachte sie Vermögen mit in die Ehe - die Verfügungsgewalt und Einkünfte aus demselben an den Mann übergingen. Ab 1958 wurde die Zugewinngemeinschaft gesetzlicher Güterstand (falls nicht anders vereinbart). Die Zugewinngemeinschaft besagt, daß während der Dauer der Ehe die Vermögen der Ehegatten getrennt bleiben, das während der Ehe ,dazugewonnene" unter den Ehegatten ausgeglichen wird. Die selbständige Verfügung beider über ihre getrennten Vermögen unterliegt allerdings Beschränkungen. Sie darf die wirtschaftliche Grundlage der Familie nicht gefährden. Ergänzend gilt zu bedenken, daß sich an der abhängigen Stellung der nichtberufstätigen Frau damit insofern nichts ändert, als sich die Zugewinngemeinschaft für sie ohnehin nur dann auswirken kann, wenn der lohnabhängige Ehemann mehr verdient, als für den Unterhalt der Familie erforderlich. In ihren ökonomischen Konsequenzen für beide Ehepartner bedenklich, für die Frau in jedem Fall erst einmal positiv ist die neuerliche Erweiterung der Zugewinngemeinschaft um Renten- und Pensionsansprüche.

13 Vgl. Habermas, Jürgen, a.a.O., S. 64

14 Zur Frage der Bezahlung der Frau durch den Lohn des Mannes: ,Wenn lange Zeit ein Teil der männlichen Arbeiter aus ihrem Lohn eine Frau unterhielt und diese nicht erwerbstätig wurde, so nicht deshalb, weil der Lohn des Arbeiters für mehrere Personen ausreichte, sondern weil er mit seinem Lohn Ökonomie betrieb. Stellte ihm die Frau not- 
duktion notwen digen Güter gekauft werden. Das bedeutet: in der Notwendigkeit ihrer eigenen Reproduktion ist ihr Interesse an der Verkaufbarkeit der Arbeitskraft ihres Mannes und damit seiner Reproduktion begründet.

Weiter kann ihre Teilnahme an den gesellschaftlichen Verkehrsformen nur über Geld stattfinden. Sie erfolgt demnach stellvertretend (15), indem die Frau die Konsumgüter realisiert, die zur Aufrechterhaltung der Arbeitskraft - einschließlich der eigenen - erforderlich sind (16). In dieser Konsumtionsfunktion realisiert sie aber gleichzeitig Kapitalwerte, denn die Umsetzbarkeit von Warenkapital in Geld ist eine notwendige Voraussetzung der Reproduktion des Kapitals.

Analysiert man die Lohnform nun unter dem Aspekt, daß dem Lohnarbeiter nur so viel zugestanden wird, wie er benötigt, um sich und seine Familie zu reproduzieren, so ist damit die ständig erneute Erfordernis des Verkaufs seiner Arbeitskraft ausgedrückt. Zu berücksichtigen, daß der lebensnotwendige Zusammenhang von Produktion und Konsumtion unter kapitalistischen Bedingungen den Lohnarbeiter dazu zwingt, die Bedingungen seiner Ausbeutung ständig von neuem zu reproduzieren, heißt zu berücksichtigen, daß die Frau als Hausfrau einen unentbehrlichen Beitrag dazu leistet. Man kann also bereits hier feststellen, daß, solange der männliche Lohnarbeiter aus den unterschiedlichsten hier nicht zu erläuternden Gründen (17) nicht in der Lage ist, sich selbst zu reproduzieren und keine gesellschaftliche Lösung für die Aufzucht von Nachkommen gefunden ist, über die Notwendigkeit von Hausarbeit für diese Gesellschaft kein Zweifel bestehen kann.

Produktion (18), Konsumtion und Reproduktion können so als allgemeine Bedingungen für eine Kategorie der Hausarbeit gefaßt werden. Sie erweisen sich in

wendige Dienstleistungen und Produkte so wohlfeil zur Verfügung, daß sie, zusammen mit den Kost- und Logiskosten für die Frau, noch unter den entsprechenden Marktpreisen lagen, so widerspricht die Tatsache, daß er eine nichterwerbstätige Ehefrau hat, nicht der Definition des Lohnes als bloßem Gegenwert für die Erhaltung der individuellen Arbeitskraft. Voraussetzung eines solchen Gebrauchs seines Lohnes ist eine spezifisch historische Situation der Frau, die die Fesselung ans Haus vorschreibt und bei den nachwachsenden Frauen ein entsprechendes Weiblichkeitsverhalten entstehen läßt. So erzeugten die überkommene Bescheidung der Frauen mit Kost und Logis als Vergütung ihrer Dienste und die ehemals hohen Preise für lebensnotwendige Güter den Schein, der Lohn umfasse prinzipiell den Unterhalt für mehrere Personen, wenn sie sich nur unter die bürgerliche Eheform gestellt hatten." Heinsohn, G./Knieper, R., a.a.O., S. $111 \mathrm{f}$.

15 Zum Begriff der stellvertretenden Funktion der Hausfrau vgl. Oakley, Ann, Women's Work, The housewife, past and present, New York 1974

16 Geschicktes ökonomisches Verhalten gehört zu den an einer Hausfrau geschätzten Fähigkeiten. Es wird nicht zuletzt dafür verantwortlich gemacht, inwieweit es der lohnarbei* tenden Familie gelingt, ihr ,Vermögen" zu vermehren bzw. ,Vermögen" zu bilden. Vgl. dazu Kahn-Freund, Otto, Einige Bemerkungen zur Entwicklung des ehelichen Güterrechts, in: Simitis, S./Zenz, G. (Hrsg.), Seminar: Familie und Familienrecht, Bd, 1, Ffm 1975

17 Hierunter fallen nicht nur Aspekte der geschlechtsspezifischen Sozialisation, sondern auch quantitative und qualitative Beanspruchung durch den Arbeitsplatz, Stand des Versorgung ssystems, das dem Haushalt zur Verfügung steht u.a.

18 Die Produktion von Nachkommen ist in der Darstellung nicht mehr weiter angesprochen worden, da die Beschränkung des Sexualverkehrs auf den ehelichen Bereich eine gewisse Wahrscheinlichkeit der Nachwuchsproduktion in sich birgt. Daß dies allerdings keine ausreichende Bedingung darstellt, dokumentiert: Heinsohn, Gunnar/Knieper, Rolf, Theorie 
ihren dargestellten gesellschaftlichen Funktionen nicht nur als allgemeiner Faktor menschlicher Produktion schlechthin, sondern durchaus kapitalistisch „formbestimmt".

\subsection{Stellenwert der unbezahlten Hausarbeit für die Formbestimmtheit der Familie}

Welcher Art ist nun diese Arbeit, die, obwohl gesellschaftlich notwendig, nicht zur Kenntnis genommen, d.h. nicht bezahlt wird?

Die Frau produziert - so wurde festgestellt - Gebrauchswerte, Gebrauchswerte für andere (19) in Form von Gütern und Dienstleistungen, die unmittelbar konsumiert werden. Der Charakter dieser Arbeit als nicht wertschaffender Arbeit ist kein Grund für ihre Nichtbezahlung. Die Ausweitung des kapitalistischen Produktionsverhältnisses ist zwar der historischen Tendenz nach absolut, hält aber weiterhin Formen aufrecht, die zwar durch die dominante Produktionsstruktur bestimmt, aber nicht unter sie subsumierbar sind. Das heißt, es lassen sich durchaus Arbeiten nennen, die - obwohl nicht kapitalistisch betrieben - bezahlt werden, so Schuster, Schneider, Koch, Chauffeur, Prostituierte etc. (20).

Allein die Tatsache, daß die Arbeit im Haus durch bezahlte Dienstkräfte leistbar war und ist, als auch die, daß vor allem die Produktion sachlicher Gebrauchswerte - die folglich als selbständige Waren zirkulieren können - dem Haus ausgelagert und der kapitalistischen Produktion subsumiert wurden, spricht gegen die in Teil 2.1 vertretene These, daß die Nichtbezahlung auf den besonderen Charakter der Arbeit als individueller Reproduktionsarbeit zurückzuführen ist, sondern legt vielmehr umgekehrt den Schluß nahe, daß der besondere Charakter von Hausarbeit nur aus der spezifischen Verbindung von individueller Reproduktonsarbeit mit unbezahlter weiblicher Arbeit zu erklären ist (21).

Das der privaten Reproduktion zugrundeliegende soziale Verhältnis ist nun aber - wie ausgeführt - kein Lohnarbeitsverhältnis, sondern die Ehe. Das Gelingen einer Ehe - so Rosenbaum - setzt „die völlige ldentifizierung der Frau mit den Aufgaben und Zielen des Mannes voraus. Nur so läßt sich das Fehlen eines eigenständigen Tätigkeitsbereiches psychisch kompensieren."(22) ldentifikation als Ausdruck

des Familienrechts. Geschlechtsrollenaufhebung, Kindesvernachlässigung, Geburtenrückgang, Ffm 1974

19 In ihrer familialen Produktion produziert die Frau zwar auch Gebrauchswerte für sich selbst,.dennoch kann man davon ausgehen, daß ihre Funktion aus Hausfrau darin besteht, Gebrauchswerte für andere zu schaffen.

20 Zur klassenanalytischen Bestimmung der Zwischen- und Mittelklassen vgl. Projekt Klassenanalyse, Materialien zur Klassenstruktur der BRD, Erster Teil, Westberlin 1973

21 Die Tatsache, daß familiale Arbeiten sich durchaus gesellschaftlich wahrnehmen lassen, stellt die Frage nach der historischen Reduktion individueller Reproduktionsaufgaben auf ein Maß, das keine Delegation von Arbeit mehr erlaubt, d.h. vom Einzelnen selbst wahrgenommen werden kann. Erst unter diesen Bedingungen wäre der von Gerstein vertretenen These zuzustimmen, daß die Nichtbezahlung der Hausarbeit auf ihren spezifischen Charakter als individueller Reproduktionsarbeit zurückzuführen ist.

22 Vgl. Rosenbaum, Heidi (Hrsg.), Familie und Gesellschaftsstruktur, Ffm 1974, S. 32 
psychischer Kompensation muß aber gleichzeitig unter dem Gesichtspunkt der verhinderten Wahrnehmung eines eigenständigen Tätigkeitsbereiches gesehen wer den. Dies als Basis einer psychischen Disposition für familiale Identifikation, denn die Entwicklung des Kapitalismus ist einerseits durch Einbeziehung der Frauen in die Produktion gekennzeichnet, die Durchsetzung seiner Gesetzmäßigkeit gegenüber der historisch vorgelagerten Gesellschaftsformation basierte aber andererseits auf einem Verdrängungsprozeß von Frauen aus gesellschaftlichen Tätigkeitsbereichen (23).

Bedingüngsmomente einer Identifikation waren für die bürgerliche Frau

- mangelnde gesellschaftliche Alternativen,

- dominierende Autoritätsstellung des Mannes, basierend auf der Verfügungsmacht über Eigentum als ökonomischer Existenzsicherung,

- soziale Sicherheit (Alter) über die Vererbung von Eigentum durch Nachkommenschaft.

Daß diese Identifikation sich mit Proklamation der bürgerlichen Freiheits- und Gleichheitsrechte als widersprüchlich darstellte, davon zeugt die bereits sehr früh entstehende ,bürgerliche“" Frauenbewegung (24).

Die Gleichsetzung der bürgerlichen Interessen mit denen der Gesellschaft schlechthin erforderte die Notwendigkeit, den eigenen Verhaltensnormen über die eigenen Klassengrenzen hinaus Geltung zu verschaffen. Die Lohnarbeiterfamilie so Heinsohn/Knieper - ist eine „Erfindung“" der bürgerlichen Gesellschaft und bedurfte direkter und in direkter staatlicher Intervention, um sie zu institutionalisieren und aufrechtzuerhalten (25). Den Identifikationsmomenten der bürgerlichen Frau fehlt hier die Basis. Die Wahrnehmung eines Tätigkeitśbereiches stellte sich hier nicht so sehr unter dem Gesichtspunkt einer Verhinderung gesellschaftlicher Alternativen (26), sondern als ökonomischer Zwang dar (27). Der damit bedrohten Familienorien-

23 „das eigentliche Novum in der Geschichte der Familie (ist) nicht die erwerbstätige Frau, sondern gerade umgekehrt die Hausfrau. ,Der Ty.p der Familie, der die Frau auf den Haushalt beschränkt und dem Mann die Rolle des einzigen Ernährers vorbehält, stellt in der Tat nur einen Übergangstyp dar' (A. Myrdal, V. Klein). Genauso können wir die steigende Erwerbstätigkeit der Frauen und vor allem ihr langsames Eindringen in verschiedene wirtschaftliche Bereiche nur als einen Prozeß der Rückgewinnung ihrer früheren wirtschaftlichen Position, allerdings unter anderen gesellschaftlichen und wirtschaftlichen $\mathrm{Be}-$ dingungen, betrachten. Dies eigentlich Neue an der ganzen Entwicklung ist nur der außerhäusliche Charakter der heutigen wirtschaftlichen Tätigkeit der Frau - der auch den gegenwärtigen Konflikt zwischen ihrer Berufs- und Mutterrolle verursacht. Erwerbstätig war die Frau schon immer." Wagnerova, Alena K., Mutter, Kind, Beruf, Reinbek b. Hamburg 1976, S. 25

24 Vgl. die Petition der Pariserinnen in der Nationalversammlung 1789, in der sie politische Gleichberechtigung und wirtschaftliche Betätigungsfreiheit des weiblichen Geschlechts forderten. Ebenso die „Declaration des droits de la Femme" der Olympe de Gouges. Dazu besonders Schröder, H./Sauter, Th., Zur politischen Theorie des Feminismus, in: Beilage zu „Das Parlament", B 48/77 vom 3.7.1977

25 Heinsohn, G./Knieper, R., Theorie des Familienrechts..., a.a.O.

26 Insofern natürlich auch Verhinderung von Alternativen, als Ausbildungsniveau, Art und Weise der Tätigkeiten und die Schwierigkeiten der doppelt arbeitenden Frauen, für eine qualitative Veränderung dieses Bereichs einzutreten, zur Folge haben, daß Frauen hier häufig nur solange arbeiten, wie es finanziell unabdingbar ist.

27 Entsprechend differierten anfänglich auch die Forderungen der gekennzeichneten ,bür- 
tierung erweist die Definition der „Zusatzarbeit" ihre nicht nur ideologischen, sondern auch materiellen Dienste, verhinderte sie mit der so begründeten geringeren Entlohnung eine mögliche materielle Eigenständigkeit der Frau (28).

Entsprechend der häufig nicht ausreichenden Versorgungsleistung des Mannes fehlt die sachliche Basis seiner Aut orität. Die Vererbung der spezifischen Form seines Eigentums bietet keinerlei Versorgungsleistung im Alter, im Gegenteil, die Nachwuchsproduktion führt zur Verschlechterung der materiellen Lebensbedingungen der Familie (29).

Das Reproduktionsinteresse des Eigentümers an Produktionsmitteln widerspricht so in seiner Familienform objektiv der Situation der lohnabhängigen Familie.

Die auf dieser Basis bereits vor rund hundert Jahren proklamierte Auflösung der Familie zeigte sich allerdings erstaunlich zählebig (30).

An dieser Stelle würde sich - im Rahmen dieser Arbeit aller dings nicht leistbar - als notwen dig erweisen, den Lohnarbeiter entsprechend seiner Stellung im gesamtgesellschaftlichen Produktions-/Reproduktionsprozeß zu differenzieren, denn sein durch die spezifische Form des Eigentums hervorgerufenes Scheininteresse als Privateigentïmer unterliegt im Grad seiner Durchbrechung objektiv dem Entwicklungsstand einer Gesellschaft, subjektiv den Bedingungen seiner Wahrnehmung. Für den individuellen Schein privater Ökonomie seien als Bedingungsfaktoren herausgegriffen: die reibungslose Verkaufbarkeit der Arbeitskraft und die Quantität des Lohnes.

Der Rahmen der privaten Ökonomie bestimmt wesentlich den Arbeitsanfall in der Familie, d.h. die Möglichkeit der Nutzung gesellschaftlicher Dienstleistungen.

Es kann angenommen werden, daß sich in lohnarbeitenden Familien mit eingeschränktem Rahmen privater Ökonomie der Arbeitsaufwand für die Frau entsprechend umfangreicher darstellt. Erstaunlicherweise oder logischerweise erweist sich aber gerade für diejenigen, die die Arbeit zwangsweise wahrnehmen müssen, die Negation der Arbeit durch die Nichtbezahlung als hergestellte Identifikation.

Um dies zu erläutern, muß darauf verwiesen werden, daß Veräußerung von Arbeit nicht nur Vergegenständlichung des Produzenten, sondern, als gesellschaftiiche, gleichzeitig produzierte Verkehrsformen beinhaltet.

Die familiale Arbeit ist keine Lohnarbeit, Charakteristikum der kapitalistischen Gesellschaft. Sie wird damit auf Gesellschaftsformationen verwiesen, die, dem Kapitalismus historisch vorgelagert, logisch in seine Voraussetzungen eingingen, $\mathrm{d} h$. in der Kateg orie des Gebrauchswertes notwendiges Moment des Kapitalismus bleiben.

gerlichen“ und „proletarischen“ Frauenbewegung. Das Recht auf Arbeit einerseits stellte sich andererseits als Schutz vor Arbeit dar.

28 Auch die Geburtenpolitik - insbesondere die Diskussion um den $\S 218$ - erweist hier ihren Stellenwert, wirkt sich die Geburt von Kindern immer wieder als Zwang zur Familiengründung aus.

29 Aus den Konkurrenzbedingungen der Arbeiterfamilie allein, wie bei Heinsohn/Knieper $\mathrm{zu}$ lesen, eine Verweigerung der Nachwuchsproduktion abzuleiten, scheint der Verfasserin dieser Arbeit nicht ausreichend. Vgl: Barabas, F./Sachße, Ch., Bevölkerungspolitik und Vergesellschaftung von Erziehung, in: „Kritische Justiz“ 2/75

30 Vgl. Engels, Friedrich, Der Ursprung der Familie, des Privateigentums und des Staates, MEW 21, Berlin (DDR), 1973 
Die Gesellschaftlichkeit der Produzenten vorkapitalistischer Gesellschaftsformationen stellte sich nicht - wie bereits dargestellt - hinter ihrem Rücken her, sondern war - aufgrund des konkret nützlichen Charakters ihrer Arbeit - unmittelbarer Ausdruck ihrer Produktion füreinander. ,In den persönlichen Verhältnissen der Produzenten in vorkapitalistischen Gesellschaften realisieren" sich so die besonderen Bedingungen ihrer Produktion; die persönlichen Verhältnisse existieren nicht ,an sich “ neben den besonderen Produktionsverhältnissen, sondern sind unmittelbarer Ausdruck derselben." $(31)$

Was bedeutet nun die Verausgabung einer solchen Arbeit in einer kapitalistischen Gesellschaft?

Die gesellschaftliche Verleugnung der Hausarbeit löst die hergestellten persönlichen Beziehungen von ihrer materiellen Basıs, der Arbeit. Die Beziehungen erscheinen so nicht mehr als Ausdruck von Arbeit, sondern bieten - so verselbständigt die Möglichkeit der Umkehrung, d.h. die Erklärung der Funktionen aus diesen Beziehungen selbst. Arbeit wurde so zur Arbeit aus Liebe.

Die Wahrnehmung eigener Leistung - nicht über die gesellschaftlich übliche Form von Geld - ; sondern über die Dankbarkeit und Zuneigung von Mann und Kindern - muß als eine psychische Disposition hervorragenden Ausmaßes angesehen werden (32). Nicht nur ist die Arbeit als solche nicht sichtbar, d.h. ,da ihre Arbeit aber nicht als Arbeit bewertet wird, fühlt sich die Hausfrau am Abend erschöpft, ohne daß sie ein Recht dazu zu haben glaubt, und so widerfährt ihr neben der Ungerechtigkeit auch noch eine Kränkung" (33), sie entzieht sich auch jeder Form der Meßbarkeit, Sexualität, Emotionalität; Putz- und Kochdienste stehen so auf einer Stufe (34). Jede Form der Verwertung oder Vernachlässigung - letzteres besonders im Falle einer Berufstätigkeit der Frau - ist mit Entzug der Anerkennung bedroht, d.h. nur auf Kosten psychischer Konflikte leistbar (35). Die üblicheren Formen des Protestes sind daher subtiler Art, d.h. Alkohol, Drogen bzw. Medikamente, psychische Erkrankungen und Formen der Überidentifikation, wie Putz-, Herrschsucht etc. (36).

Die Frage nach der Ursache dieser von Dalla Costa bezeichneten ,Kastration“ der Frau verweist letztendlich auf den Inhalt der im Haus geleisteten Arbeit. Hier zeigt sich der notwen dige Zusammenhang von familialer ldentifikation und nichtentfremdeter Arbeit. „Die Tatsache, daß der Arbeiter gezwungen ist, seine Arbeitskraft gegen Geld zu verkaufen, schließt notwendig die Entfremdung des Arbeiters von seiner bestimmten Tätigkeit ein. Die bestimmte Tätigkeit ist ihm prinzipiell nur Mittel zur Erlangung eines Wertquantums. Nicht das, was der Arbeiter weggibt, sei-

31 Ramshorn, Angelika/Engeler, Christiane, Unveröffentlichtes Manuskript, S. 11

32 Die Hilfeleistung geschlechtsspezifischer Sozialisation sei hier nicht unerwähnt.

33 Riesmann, David/Denney, Reuel/Glazer, Nathan, in: Wagnerova, Alena K., a.a.O., S. 18

34 „Liebe“ geht ja bekanntermaßen durch den Magen.

35 Die Rollenkonflikte werden häufig mit Begriffen der Schizophrenie-Forschung gefaßt, so die ,double bind"-Situation. Vgl. Brandt, Gisela u.a., Zur Frauenfrage im Kapitalismus, Frankfurt 1973

36 Vgl. u.a. Chesler, Phyllis; Frauen - das verrückte Geschlecht? Reinbek b. Hamburg 1974, Friedan, Betty, Der Weiblichkeitswahn oder die Selbstbefreiung der Frau, Reinbek b. Hamburg 1970 
ne bestimmte Arbeit, sondern das, was er erhält, die Geldsumme, mit der er seine Reproduktion sichern kann, ist für ihn primär wichtig. Die Identifikation mit dem bestimmten Inhalt der Tätigkeit (...) tritt von vorneherein zurück hinter dem Interesse an der klingenden Münze. Auf seiten des Arbeiters ist also auch in diesem Verhältnis die objektive Gleichgültigkeit gegen den bestimmten Inhalt seiner Tätigkeit gesetzt und daher auch immer als Bewußtseinstatsache vorhanden (...). Die Herausbildung dieser Gleichgültigkeit gegen den bestimm ten Inhalt der Tätigkeit macht es dem Arbeiter der Tendenz nach möglich, eine Differenz zwischen der konkret-nützlichen Tätigkeit und ihrer gesellschaftlichen Form, der Arbeit, der Arbeit zur Reproduktion des bloßen Arbeitsvermögens, d.h. der Lohnarbeit, überhaupt zu denken." (37) Von dieser Tendenz sind auch Arbeiten nicht ausgeschlossen, deren Produkte gemäß ihrer natürlichen Beschaffenheit nur in Form von Diensten existieren. Gleichgültigkeit als ein der Identifikation entgegenwirkendes Moment muß also - von der Funktionalität des Systems her gesehen - dem familialen Zusammenhang ausgeschlossen bleiben, um die Qualität der Arbeit zu gewährleisten, die, in der Form kapitalistischer Lohnarbeit nicht leistbar, den menschlichen Träger der Ware Arbeitskraft ausmacht, nämlich seine emotionale und psychische Produktion/Reproduktion (38).

Wurde bisher festgestellt, daß die Frau mit der Wahrnehmung ihrer familialen Arbeit einen wesentlichen Beitrag dazu leistet, den Lohnarbeiter in die physische und psychische Lage zu versetzen, die Bedingungen seiner eigenen Ausbeutung ständig von neuem reproduzieren zu müssen, so kann hier weiter präzisiert werden, daß sie so gleichermaßen die Bedingungen ihrer eigenen Ausbeutung produziert/reproduziert.

Die Herstellung unmittelbar menschlicher Beziehungen über den Akt der gemeinschaftlich füreinander realisierten Produktion ist, aufgrund des spezifischen Charakters kapitalistischer Produktion, nur noch als familialer Restbestand auf Basis der direkten Abhängigkeit der Frau möglich, abstrahiert so ständig von den menschlich bedeutsamen Qualitäten familialer Lebensform.

Die Notwendigkeit der Negation familialer Arbeit durch ihre Nichtbezahlung erweist so ihren Legitimationscharakter hinsichtlich folgen der Tatbestände:

- der Verschleierung eines dem Kapitalismus wịdersprechenden direkten Abhängigkeitsverhältnisses,

-- der Verleugnung von Arbeit als materieller Basis menschlicher Beziehungen,

- der Verschleierung von Arbeitsbedingungen, die nicht den kapitalistischen Kriterien von Rationalität entsprechen.

37 Projekt Klassenanalyse, Materialien zur Klassenstruktur der BRD, a.a.O., S. $281 \mathrm{f}$.

38 Zum Problem der ,, tendenziellen Gleichguiltigkeit" des Lohnarbeiters hinsichtlich der Arbeit am Menschen, vgl. Müller, Ludmilla, Die Wertlosigkeit der Arbeit der Kinderaufzucht im Kapitalismus - wertlose Arbeit: Über die Folgen der Nichtbewertung der Arbeit der Mütter für das Bewußtsein der Frauen als Lohnarbeiterinnen, in: Prokla 22/76 


\subsection{Probleme einer Ableitung des Lohns für die Hausarbeit}

An dieser Stelle soll die Argumentation noch einmal zusammengefaßt werden. Den Vertreterinnen der Forderung nach Lohn für die Hausarbeit selbst ist aufgrund ihres methodischen Vorgehens die Möglichkeit einer Ableitung der Lohnforderung verwehrt. Die Versuche, die Forderung dennoch als berechtigt zu reflektieren, haben bisher zu den folgenden Ergebnissen geführt.

Die Hausarbeit ist Arbeit, aber keine mehrwertschaffende Arbeit, Obwohl gesellschaftlich notwendige Arbeit, besteht ihre ,kapitalistische Formbestimmtheit“ in der Negation dieser Arbeit. Sie wird damit auf die Stufe vorkapitalistischer Produktion verwiesen. Die Probleme, die sich so für eine Ableitung des Lohns für die Hausarbeit ergeben, liegen auf der Hand. Obwohl Arbeit, fehlt ihr der Wertausdruck, dargestellt im Geld, d.h. sie kann sich als private Arbeit nicht gesellschaftlich darstellen.

Weil sie sich gesellschaftlich nicht darstellen kann, entzieht sich die private Arbeit den gesellschaftlichen Bewertungsmöglichkeiten.

Auf dieser Ebene bleibt nur die Flucht in den Widerspruch der „Natürlichkeit“ der dennoch gesellschaftlich formbestimmten Familie (39), oder aber - der Bewegung „Lohn für die Hausarbeit" folgend - das Verlassen des bisher akzeptierten Rahmens der Diskussion.

Im folgenden soll diese Problematik beispielhaft an einigen Ansätzen, die Hausarbeit als Arbeit zu begreifen versuchen, dargestellt werden (40).

Der bei Ludmilla Müller (41) in Kritik an Engels entwickelte Arbeitsbegriff versucht, einen Bereich familialer Arbeit - die Kinderaufzucht - seiner naturhaften Bestimmung zu entziehen und als Arbeit begreifbar zu machen. Arbeit wird von Müller verstanden als „das planvolle Verändern der in der Natur gegebenen Formen der Dinge“ (42). Die Unterscheidung von Natur ,das Austragen und das Gebären eines Kindes sind Mühe, Anstrengung und Schmerzen, aber sie sind nicht Arbeit, sondern Naturvorgang (der allerdings immer auch gesellschaftlich mitgeprägt ist und entsprechend von der Frau eine aktive Stellungnahme verlangt - die Geburt geschieht nicht nur wie beim Tier) " und Arbeit „,dem menschlichen Umformen der Naturgegenstände, der Arbeit im engeren Sinn" (43) bleibt unscharf (44).

39 Vgl. Women and Revolution, Dalla Costa/James and the Subversion of Marxism..., a.a.O., S. $44 \mathrm{ff}$.

40 Die Vefrtreter/innen đer dargestellten Ansätze fordern selbst keinen Lohn für die Hausarbeit. Die Auseinandersetzung um die Anerkennung von Hausarbeit ist allerdings notwendige Voraussetzung einer möglichen Lohnforderung.

41 Ludmilla Müller, Kinđeraufzucht im Kapitalismus - wertlose Arbeit..., a.a.O.

42 ebenda, S. 17

43 ebenda, S. 17

44 In einem an der Sommeruniversität Berlin 1976 vorgetragenen Arbeitspapier zur Arbeit von Ludmilla Müller äußern die Verfasserinnen die nicht unberechtigte Befürchtung, daß die Unschärfe der Begrifflichkeit zu einer Unterbewertung der Hausarbeit führen könnte, zumal - hier nehmen sie Bezug auf die bereits zitierte Arbeit von Ann Oakley -- Kinder in erheblichem Ausmaß zur Ausweitung der Hausarbeit beitragen. Die Verfasserinnen sind entgegen Müller der Meinung, daß die Unterscheidung zwischen Natur und Arbeit 
Entsprechend (steht) ,, die ,Arbeit am lebendigen Produkt", die Tätigkeit der Kinderaufzucht, (...) auf halbem Wege zwischen natürlichen Entstehungsprozessen und dem menschlichen Umformen der Naturgegenstände, die Arbeit im engeren Sinn." (45)

Konsequenzen hinsichtlich einer notwendigen gesellschaftlichen Anerkennung von Hausarbeit zieht Müller nicht. Sie bleibt selbst in dem von ihr konstatierten Widerspruch der bürgerlichen Gesellschaft befangen, indem sie einerseits davon ausgeht, daß ,die Arbeit der Frauen im Haushalt und an den Kindern (im Kapitalismus, A. d. V.) zur wertlosen, nicht produktiven Arbeit, die keine unmittelbare oder auch mittelbare Bedeutung für die Produktion von Wert und Mehrwert hat "(46), andererseits aber feststellt, daß die Frauen mit dieser Arbeit ,fundamentale Voraussetzungen ,für das Funktionieren der gesellschaftlichen Lebenshaltung im Kapitalismus" schaffen (47). Mit einer Kritik an der Marxschen Wertbestimmung der Ware Arbeitskraft tragen Engeler/Ramshorn (48) zur Diskussion bei. Es ist korrekt, so die Verfasfasserinnen, den Wert der Ware Arbeitskraft gleich dem jeder anderen Ware durch die zu seiner Produktion bzw. Reproduktion notwendige Arbeitszeit zu bestimmen (49).

Es ist weiterhin korrekt, davon auszugehen, daß die Arbeitskraft als Anlage des lebendigen Individuums einer gewissen Summe von Lebensmitteln bedarf, um sich zu erhalten bzw. zu reproduzieren.

Es ist nicht korrekt, ,die in der gesellschaftlichen Produktion dieser Lebensmittel verausgabte Arbeit (die sich als bestimmbare Wertgröße fassen läßt) gleichzusetzen mit der zur Reproduktion der Arbeitskraft insgesamt notwendigen Arbeit “ (50), denn „die zur umf assenden Reproduktion der Arbeitskraft verausgabte Arbeit bestimmt sich aber durch Verausgabung von Arbeit im Produktions- sowie Reproduktionsbereich" (51).

nicht nachzuvollziehen sei, da auch natürliche Prozesse der menschlichen Einwirkung bedürften. Die Reproduktion der Gattung könne nicht als allgemeiner, überhistorischer Prozeß gefaßt werden, da es sich zwar um Prozesse handele, die sich in ihrer Grundstruktur in allen Gesellschaften wiederholen, in der konkreten Ausprägung jedoch je nach der Entwicklung der Produktionskräfte und nach Art der Verausgabung der Arbeit verschieden seien.

Müller, Ludmilla, a.a.O., S. 17. Es muß ergänzt werden, daß Müller sich in einem Nachwort zum Abdruck der von ihr 1972 verfaßten Diplomarbeit in der „Prokla“ den Auffassungen der Verfasserinnen des Arbeitspapiers annähert. Dies als Resultat eines Diskussionsprozesses mit der Redaktionskonferenz der "Prokla“, in der Müller genau mit dem Argument konfrontiert wird, daß die Reproduktion der Gattung eher als allgemeiner überhistorischer Prozeß zu verstehen sei. Vgl. „Prokla“ 22/76, S. 61

46 ebenda, S. 55

47 ebenda, S. 56. Vgl. das bereits aufgeführte Arbeitspapier der Sommeruniversität Berlin 1976

48 Ramshorn, Angelika/Engeler, Christine, unveröffentlichtes Manuskript

49 Die Verfasserinnen beziehen sich hier auf Marx, Karl, Das Kapital II, a,a.O., S. 185

50 Zitat von Marx, auf das sich die Verfasserinnen beziehen: ,Die zur Produktion der Arbeitskraft notwendige Arbeitszeit löst sich also auf in die zur Produktion dieser Lebensmittel notwendige Arbeitszeit..." Marx, Karl, a.a.O., S. 185

51 Ramshorn/Engeler, a.a.O., S. 5 
Soweit gesellschaftlich produziert, gehen diese Arbeiten in den Wert der Ware Arbeitskraft ein. Da die Arbeit der Frau im individuellen Reproduktionsbereich aber keine warenproduzieren de Arbeit ist - insofern auch keine Ware schafft „kann sie in die Bestimmung des Werts der Ware Arbeitskraft nicht eingehen. Wenn Marx also den Wert der Ware Arbeitskraft bestimmt, ohne die Reproduktionstätigkeit der Frau miteinzubeziehen, ist dies korrekt, es ist aber nicht korrekt, die zur Erhaltung des Individuums gesellschaftlich notwendige Arbeit gleichzusetzen mit der Arbeitszeit, die zur Produktion der in der individuellen Reproduktion eingehenden Waren notwendig ist."

Leider führen die Verfasserinnen die Konsequenz dieser Annahme hinsichtlich einer möglichen positiven Arbeitsbestimmung nicht aus. Für unseren Zusammenhang bleibt hier nur zu schließen, daß die Frau arbeitet, mit ihrer Arbeit aber nicht zur Wertbestimmung der Ware Arbeitskraft beiträgt.

Diese Argumentation vertritt auch - wie bereits ausgeführt - Gerstein (52). Es bleibt hier allerdings zu ergänzen, daß sie die Einbeziehung der Hausarbeit in die Wertbestimmung der Ware Arbeitskraft nicht für undenkbar hält, für diese Möglichkeit allerdings nur die Reproduktionsarbeit der Ware Arbeitskraft in Betracht zieht, nicht die Produktions- und Reproduktionsarbeit zukünftiger Arbeitskraft - also die Kinderaufzucht.

$\mathrm{Zu}$ einer Doppelbestimmung der Hausarbeit gelangt der bisher umfassendste Ansatz (53).

Hausarbeit, soweit sie gesellschaftliche Gebrauchswerte hervorbringt, wir dunterschieden ,in diejenigen Tätigkeiten, die ein selbständiges sachliches Arbeitsprodukt schaffen, das von Mann und Kindern konsumiert wird (Zubereitung und Beschaffung von Lebensmitteln, lnstandhal tung von Kleidung und Wohnung) "(54) und ,in diejenigen Tätigkeiten, die kein selbständiges sachliches Arbeitsprodukt hervorbringen, sondern Dienstleistungen sind ... (körperliche Bildung, Geburt und Aufzucht der Kinder, Krankenpflege, primäre Sozialisation)" (55).

Betrifft die erste Art der Tätigkeit durch deutliche Trennung von Arbeitsprodukt und Konsument ,zunächst nur das ökonomische Verhältnis von Mann und Kindern auf der einen, der Frau auf der anderen Seite" (56), so muß die zweite Art der Tätigkeit - aufgrund des Zusammenfallens von Konsument und Arbeitsprodukt - unter zweierlei Gesichtspunkten betrachtet werden, einerseits - soweit Mann und. Kinder Konsumenten - unter bereits oben genanntem ökonomischen Verhältnis, an dererseits, ,im ökonomischen Verhältnis zwischen Kapitalist und Frau, nämlich sofern Mann (und Kinder) Arbeitsprodukt der Frau und dieses Arbeitsprodukt selbst wichtigstes Produktionsmittel des Kapitals ist, also sofern die Frau in dem Mann (und Kindern) Gebrauchswerte für das Kapital schafft" (57). Das ,ökonomische“"

\footnotetext{
52 Vgl. Teil 2.1 dieser Arbeit

53 Perschke, Rolf-Dieter, Zum Lohn für die Hausarbeit, 1975, unveröffentlichtes Manuskript

54 ebenda, S. 9

55 ebenda, S. 9

56 ebenda, S. 9

57 ebenda, S. $9 \mathrm{f}$.
} 
Verhältnis zwischen Mann und Frau ist aber kein Arbeitsverhältnis, Der Mann bezahlt der Frau keinen Lohn, weder die Arbeitskraft der Frau, noch ihre Produkte nehmen - obwohl gesellschaftliche Gebrauchswerte - die Form von Waren an. Daraus folgt ,im ökonomischen Verhältnis zwischen Mann und Frau zählt also die Hausarbeit nicht als bürgerliche Arbeit" $(58)$. Im ökonomischen Verhältnis zwischen Frau und Kapitalist konsumiert letzterer zwar die von der Frau produzierte Ware, die Frau erscheint aber nicht als Produzentin dieser Ware, noch erhält sie den Wert dieser Ware in Geldform. Da diese Arbeit aber als tauschwertsetzende Arbeit erscheint, ist die Arbeit der Frau bürgerliche Arbeit. Da aber nicht sie den Wert der Ware Arbeitskraft bezahl t bekommt, ,kann die Rolle der Frau nicht die eines einfachen Warenproduzenten sein" (59). Da sie auch nicht den Wert ihrer Arbeitskraft vom Kapitalisten bezahlt bekommt, „kann ihre Rolle auch nicht die eines Lohnarbeiters sein" (60). Es folgt, ,im ökonomischen Verhältnis zwischen Frau und Kapitalist wird zwar offenbar, daß die Hausarbeit bürgerliche Arbeit ist (...), aber nicht für die Frau“" (61), d.h. sie erscheint ihr nicht als solche.

Der Ansatz Perschkes bietet die Möglichkeit, eine Lohnforderung hypothetisch durchzuspielen;

1. ökonomisches Verhältnis Mann-Frau:

der Mann bezahlt aus seiner Revenue die Güter/Dienste oder die Arbeitskraft der Frau. In erstem Falle wäre die Frau als selbständige Produzentin anzusehen, d.h. sie besäße ihre eigenen Produktionsmittel, im letzten Fall als Dienstbotin.

2. ökonomisches Verhältnis Kapitalist-Frau:

der Kapitalist kauft die Ware Arbeitskraft Mann als Produkt der Frau. In dem Fall käme der Lohn des Mannes der Frau zu. Auch hier träte sie als selbständige Produzentin auf, die sich in diesem Fall aber gegen variables Kapital austauscht. Der Kapitalist kauft die Arbeitskraft der Frau, damit sie die Ware Arbeitskraft Mann reproduziert. Hier wäre ihre Arbeit - als werterhaltende - noch einmal unter dem Gesichtspunkt der produktiven Arbeit zu diskutieren.(62).

Die Annahmen erscheinen absurd.

Auf dieser Ebene bleibt so vorläufig nur zu konstatieren: entweder, die Lohnforderung muß als ungerechtfertigt angesehen werden, womit allerdings nach wie vor die Tatsache besteht, daß gesellschaftlich notwen dige Arbeit unbezahlt geleistet wird,

oder aber das angewandte Kategoriensystem muß dahingehend hinterfragt werden, inwieweit es auf Basis der entwickelten Kategorien überhaupt in der Lage ist, die Arbeit sichtbar werden zu lassen.

\footnotetext{
58 ebenda, S. 10

59 ebenda, S. 11

60 ebenda, S. 11

61 ebenda, S. 11

62 Wo, bei diesen Annahmen, die Kinderaufzucht einzuordnen wäre, ist nicht ganz ersichtlich.
} 
Die Untersuchung der Hausarbeit bedarf - wie vor allem die Probleme des letzten Teils gezeigt haben - der Weiterführung. Da Hausarbeit als ein wesentliches, wenn nicht zentrales Element der weiblichen Rolle angesehen werden muß, ist es nicht gerechtfertigt, diese Kategorie zu vernachlässigen. Hier wäre auf eine Auseinandersetzung mit Theorie und Praxis sozialistischer Frauenemanzipation zu verweisen.

Daß die Hausarbeit einer nähẹren Untersuchung bedarf, zeigt auch die für die Bundesrepublik mit dem Gleichberechtigungsgesetz von 1957 feststellbare Tendenz. die Hausarbeit gleichwertig neben die Erwerbstätigkeit zu stellen (63).

Für diesen - weil von allen Prinzipien überprüfbarer Leistungsmessung bewußt absehend - von Heinsohn/Knieper so bezeichneten Definitinsakt (64) liegen mit der seit neuestem erschienenen Studie zur Arbeitsbewertung in der Hauswirtschaft Kriterien als Arbeitsbemessungsgrundlage vor (65). Daß der Wert von Hausarbeit durchaus auch vorher in Geld meßbar war, zeigen versicherungsrechtliche Fälle von Schadensersatzansprüchen bei Unfall oder Tod der Hausfrau (66).

Was seitdem unter Gleichwertigkeit der Hausarbeit verstanden wurde, reicht von - die geschlechtsspezifische Arbeitsteilung legitimierender - Gleichheit bei biologischer und funktioneller Unterschiedlichkeit (67) bis hin zur neuesten Wahlfreiheit, d.h die Ehegatten regeln die Haushaltsführung in gegenseitigem Einvernehmen (68).

Konsequenterweise müssen sich Eheleute bei gleicher beruflicher Inanspruchnahme auch in gleichem Umfang an der Hausarbeit und der Kinderbetreuung beteiligen (69).

Was drückt diese Entwicklung nun aus? Zweifelsohne muß sie als Verallgemeinerung des Lohnarbeitsverhältnisses, der dazu in Relation stehenden Herstellung der Rechtssubjektivität der Frau und damit auch einer tendenziellen Auflösung der be-

63 Vgl. dazu Heinsohn/Knieper, a.a.O., S. 122/125

64 ebenda, S. 126

65 Anhand einer Reihe von Testhaushalten wurde festgestellt, welche Tätigkeiten in den meisten Haushalten immer wieder vorkommen. Quantitative und qualitative Faktoren (Zeit, Fähigkeiten, Umgebungseinflüsse) wurden mathematisch zueinander in Verbindung gesetzt und in Punktwerten ausgedrückt. Der Umsetzung dieser Punktwerte in Geldwerte wurden die in Beziehung.zueinander gesetzten Gehälter aller in Hauswirtschaft, Krankenpflege und Erziehung tätigen Personen zugrundegelegt. Der Lohn für Hausarbeit läge entsprechend zwischen 1500 und 3000 DM monatlich.

Vgl. Deist/Böhner, Arbeitsbewertung in der Hauswirtschaft, München 1977

66 Vgl. ,Eltern“ Nr. 10 vom Oktober 1976, S. 34

Frankfurter Rundschau vom 3. Februar 1977, 5. Februar 1977, 5. März 1977

Die in diesen Fällen zu erstattenden Leistungen lagen zwischen 100 und 1425 DM monatlich.

67 Junker, Reinhold, Die Lage der Mütter in der Bundesrepublik Deutschland, Ffm 1965, Bd. 1 - 3. Sie ist vor allem deshalb erwähnenswert, weil sie unter anderen die Vorlage für die erste Frauenenquete der Bundesregierung 1966 bildete.

68 Vgl. Neuformulierung des $\$ 1356$ BGB im neuen Ehe- und Familienrecht.

69 Urteil des Bundessozialgerichts, in: Frankfurter Rundschau vom 21. Januar 1977 
stehenden Familienform gesehen werden (70).

Was heißt aber Wahlfreiheit der Geschlechter? Sie setzt voraus, daß beide Geschlechter gleiche Ausgangsbedingungen und gleiche Entscheidungsmöglichkeiten haben. Es muß nicht im einzelnen auf die für Kinder nach wie vor zuständige Frau, die ,Partnerschaft" bezüglich der Aufgabenverteilung in der Ehe, auf die Beschäftigungsstruktur weiblicher Arbeitskräfte, größere Arbeitsplatzunsicherheit, Qualifikationsdefizite, Lohndiskriminierung, Unterrepräsentiertheit in qualifizierten Berufen etc., etc. hingewiesen werden, um diese Wahlfreiheit als Schein zu entlarven.

Die Frage nach der Möglichkeit einer Aufhebung dieser aktuellen ,Mängel“" stellt die Frage nach den strukturellen Grenzen einer Wahlfreiheit auf Basis eines Systems, das kein Recht auf Arbeit kennt, im Gegenteil in zunehmendem Maße zu einer Freisetzung von Arbeitskräften tendiert, des weiteren - wie bereits erwähnt in doppelter Weise von der spezifischen Ausbeutung der Frau profitiert; nicht nur durch die unbezahlte Übernahme von Versorgungsleistungen an Mann und Kindern, auch durch die im Falle ihrer Berufstätigkeit billige, flexible und anpassungsfähige Arbeitskraft Frau.

Wie ist diese Entwicklung weiterhin vorstellbar (und nur auf dem Hintergrund ist eine Forderung nach Lohn für die Hausarbeit einzuordnen und eine mögliche „Institutionalisierung“ zu überprüfen)?

1. Bei abnehmender Beschäftigungszahl oder zeitlich veränderter Arbeitsorganisation zunehmende geschlechtsspezifische Konkurrenz?

Dies ist nur möglich:

- auf Basis des Verzichts von Kindern

- bei Ausführung der Hausarbeit durch je einen Partner

- Verlagerung der bisherigen Doppelbelastung der Frau auf beide - Mann und Frau

- durch gesellschaftliche Übernahme der Hausarbeit

2. Verweisung der Frauen auf ihre Hausfrau- und Mutterfunktion?

Letzteres erscheint - der historischen Entwicklung entsprechend - längerfristig unwahrscheinlich, zumal ein Großteil der weiblichen Arbeitskräfte einen ,unersetzbaren" Bestandteil des außerhäuslichen Arbeitsbereichs darstellen.

Kurzfristig scheint dies jedoch nicht ausgeschlossen, berïcksichtigt man, daß eingeschränkte gesellschaf tliche Alternativen nach wie vor als Grund für einen ,Rückzug" in die Familie angesehen werden müssen (71).

Welcher Weg auch immer, das sich anbahnende Konfliktpotential ist unübersehbar.

Zusammenfassend kann gesagt werden: Die Entwicklung bedarf - um Aussagen über den Auflösungsgrad der Familie und die Bewußtseinssituation der Frau zu erlauben - der ergänzenden Untersuchung von Hausarbeit, u.a. hinsichtlich

70 Vgl. Heinsohn/Knieper, a.a.O.

71 Vgl. Traudel Lucius, Die Auswirkungen der Arbeitosigkeit auf die Situation proletarischer Mädchen, in: „links“ 77/76. Vgl. Florence Hervê/Renate Wisbar, Rückwirkungen der Wirtschaftskrise auf die Situation der Frauen in der BRD, in: Blätter für deutsche und internationale Politik, 8/76 
- ihrer historischen Entstehung

- - ihrer historischen Funktionsveränderung

- ihres Umfangs und Grads der Wahrnehmung durch die Frau auf Basis der gekennzeichneten Differenzierungen des Lohnarbeiters

- ihrer Verän derung durch eine Berufstätigkeit der Frau

- der gesamtgesellschaftlichen Strukturveränderung von individuellem und gesellschaftlichem Produktions-, Reproduktionsbereich.

Hausarbeit muß als eine Kategorie der bürgerlichen Gesellschaft angesehen werden. Sie entstand im Zuge der Industrialisierung und muß als notwendige Reaktion auf die Veränderung der Organisationsstruktur der Arbeit in der Fabrik und die Zerstörung der vorkapitalistischen Familienform als Organisationsform der produktiven Arbeit gesehen werden. Im Mittelpunkt stand das Problem einer Aufzucht von Kindern, die entsprechend der sozio-ökonomischen Veränderung aus dem Produktionszusammenhang der Eltern herausfielen. Die Hausfrauenrolle war neben den bereits erwähten direkten und indirekten staatlichen Interventionen (72) das Produkt einer politisch-ideologischen Kampagne (73). Der Entstehungsprozeß war von Anfang an befangen in den Widersprüchen der bürgerlichen Gesellschaft, d.h. stand in einer komplexen Wechselwirkung mit der sich gleichzeitig nur durch Einbeziehung der Frauen in die Produktion möglichen Ausweitung der Lohnarbeit. Diese Widersprüche fanden ihren Ausdruck in der Konstituierung des familialen Zusammenhangs und bildeten die Basis seiner zunehmenden Disfunktionalität. Daß die Kategorie der Hausarbeit überhaupt als solche in das ,öffentliche "Bewußtsein gedrungen ist, muß als Verallgemeinerung der Lohnarbeit angesehen werden. Daß diese Entwicklung wesentlich auf Kosten doppelt arbeitender Frauen gelaufen ist, kann auch nicht unerwähnt bleiben. Denselben auf dieser Basis mangelndes oder rückständiges politisches Bewußtsein vorzuwerfen, muß als fundamentale Unkenntnis des gesamten Lebenszusammenhangs von Frauen angesehen werden.

Die - in der bisherigen Emanzipationskonzeption - schwerpunk tmäßige Verlagerung auf den Untersuchungsstand der außerhäuslichen Arbeit mag sich unter historischen Gesichtspunkten als vorrangig dargestellt haben, hat sich jedoch von ihren Erklärungszusammenhängen her als nicht ausreichend gezeigt und entbehrt damit ihres Ausschließlichkeitsanspruchs.

72 Heinsohn, G./Knieper, R., a.a.O.

73 Vgl. dazu

- Oakley, Ann, a.a.O.

- Duden, Barbara, Das schöne Eigentum, Zur Herausbildung des bürgerlichen Frauenbildes an der Wende vom 18. zum 19. Jhdt., in: „Kursbuch“ 47, Berlin 1977

- Bock, Gisela/Duden, Barbara, Arbeit aus Liebe - Liebe als Arbeit, Zur Entstehung der Hausarbeit im Kapitalismus, in: Frauen und Wissenschaft, Berlin (West) 1977

- Welter, Barbara, The Cult of Womanhood 1820 - 1860, in: American Quarterly 18 (1966), Part I, p. 151 - 174 


\section{Lohn für die Hausarbeit als Strategie der Frauenbewegung}

Für die strategische Ausrichtung der Frauenbewegung sei abschließend der Stellenwert einer Lohnforderung diskutiert. Die Hausarbeit - so war festgestellt worden ist gesellschaftlich notwendige Arbeit. Daß sie nicht bezahlt wird, macht ihren systemfunktionalen Stellenwert aus, d.h. der Widerspruch von gesellschaftlich notwendiger Hausarbeit und ihrer Nichtbezahlung entspricht der Logik dieses Systems.

Was würde eine Bezahlung der Hausarbeit hier bewirken? Die Hausarbeit wäre kapitalistische Lohnarbeit mit allen der Entfremdungsproblematik implizierten Konsequenzen, zudem auf einem kapitalistischer Rationalität widersprechenden hohen Kostenniveau, zu deren Minimierung mit großer Wahrscheinlichkeit eine gesellschaftliche Einflußnahme auf den ,Privatbereich" angenommen werden kann.

Der Familie als ,Hort bürgerlicher Privatheit ${ }^{\star}$ wäre so zwar endgültig der ohnehin brüchige Boden entzogen, dies läßt jedoch keinerlei voreilige Aussagen über ihre Auflösung zu (1).

Das heißt, mit der von den Vertreterinnen der Lohnforderung formulierten Annahme, daß die gesellschaftliche Anerkennung der Hausarbeit gleichzeitig eine Ablehnung der Hausarbeit durch die Frauen bedeutet (2), abstrahieren sie in ähnlichem Maße familiale Identifikationsmomente wie die von ihnen kritisierte Strategie der außerhäuslichen Arbeit, was in beiden Fällen zu einer strategischen Überschätzung der ökonomischen Selbständigkeit der Frau führt.

Das Aufbrechen der Mystifikation der Haùsarbeit - der Gleichsetzung der Frau mit ihren familialen Funktionen - setzt eine nicht nur im Geld sichtbare, sondern auch erfahrbare Ebene der Distanzierung voraus, dies um so wesentlicher aufgrund der komplexen Verwobenheit familialer Funktionen mit emotionalen Bedürfnissen.

Der von den Vertreterinnen der Lohnforderung hier erhoffte revolutionäre Weg einer Aufhebung der Hausarbeit mithilfe des Lohnes wird sich so einreihen müssen in die „Niederungen" der historisch langwierigen und mühevollen politischen „Kleinarbeit", deren Rahmen Dalla Costa folgendermaßen steckt: „Die Herausforderung der Frauenbewegung liegt darin, Kampfformen zu finden, die während sie die Frau vom Haus befreien, auf der einen Seite eine doppelte Knechtschaft der Frau vermeiden und auf der anderen Seite eine weitere Stufe der Kontrolle und Disziplinierung durch das Kapital verhindern. Dies ist für die Frauenbe wegung letztlich die Trennungslinie zwischen Reformismus und revolutionärer Politik. "(3)

Inwieweit eine Lohnfortzahilung zur Institutionalisierung, besser zu einer gesellschaftlich anerkannten Institutionalisierung (denn, hier kann den Vertreterinnen der Lohnforderung nur zugestimmt werden, die Hausarbeit ist institutionalisiert) führt, ist schwer abschätzbar. Die Wahrnehmung von Hausarbeit als Arbeit - die These soll noch einmal wiederholt werden - muß als Ausdruck der Verallgemeine-

1 Vgl. Barabas, F./Sachße, Ch., Bevölkerungspolitik und Vergeselischaftung von Erziehung, a.a.O.

2 Vgl. die Einleitung dieser Arbeit.

3 Dalla Costa, M./J ames, S., Die Macht der Frauen, a.a.O., S. 58 
rung von Lohnarbeit gesehen werden, d.h. die Einbeziehung der familialen Arbeit in die gesellschaftliche Produktion/Reproduktion wird zur Verstärkung dieser Tendenz beitragen. Die politisierende oder integrierende Wirkung einer Lohnzahlung kann nur auf dem Hintergrund dieser sich als widersprüchlich gestaltenden Entwicklung beurteilt werden.

Der Integrationsmomente wegen allerdings die Lohnforderung abzulehnen, scheint der Verfasserin nicht angemessen. Stellt die Frage nach der Berechtigung dieser Forderung die nach der von bezahlter Arbeit überhaupt, so würde die Furcht vor Integration durch die Bezahlung von Arbeit eine Ablehnung jeglicher Form ökonomischer Verbesserungen aufgrund dieses ihres integrationistischen Gehalts nach sich ziehen. Die Notwendigkeit der Bezahlung von Hausarbeit anzuerkennen heißt aber nicht, den Alleinvertretungsanspruch der Vertreterinnen dieser Strategie zu akzeptieren. Als Ausdruck von Verallgemeinerung der Lohnarbeit reflektiert diese Strategie sowohl die Widersprüche, die die Einbeziehung von Frauen in die Produktion auch für den familialen Bereich mit sich bringt, als auch die Grenzen der Strategie der außerhäuslichen Arbeit, deren emanzipatorische Hoffnung aus dieser Entwicklung der Einbeziehung der Frauen in die Produktion eine erhebliche Beschränkung durch die familiale Belastung und Identifikation der Frau erfährt (4).

Eine Strategie „Lohn für Hausarbeit" erweist hier ihren notwendig ergänzenden Charakter. Es gilt so für beide Strategien, ihren bisher alternativ diskutierten Charakter aufzugeben, und sich als ergänzend zu begreifen-(5), dies umso mehr zu einem historischen Zeitpunkt, in dem Verdrängungsprozesse aus dem außerhäuslichen Arbeitsbereich eine Aufwertung der Familie als durchaus denkbar erscheinen lassen (6).

Es bleibt, den Realitätsgehalt einer Forderung' nach Lohn für die Hausarbeit zu überprüfen.

Um eine ungefähre Vorstellung von den zu erwartenden Lohnforderungen zu vermitteln, sei der Einfachheit halber nur die Zahl der verheirateten Frauen von 15.4 Millionen herausgegriffen'(7) und eine Entlohnung von 1000.- DM (netto)

4 Vgl. hierzu besonders Müller, Ludmilla, Einbeziehung der Frauen in die Produktion als Weg zur Emanzipation? in: Blätter für deutsche und internationale Politik, 9/73

5 Die Verfasserin hält das bisher u.a. trennende Argument, daß entsprechend der sozioökonomischen Gegensätze nicht alle Frauen in eine Strategie miteinbezogen werden können, angesichts der sozialstrukturellen Veränderungen hinsichtlich der Verallgemeinerung von Lohnarbeit für kein ausschlaggebendes mehr.

6 Es sei hier auf die Kommentierung der Studie über die Arbeitsbewertung von Deist/Böhmer in „Eltern" 10/76 hingewiesen. Dort kann man lesen: ,Hausarbeit ist ein vollgültiger, werteschaffender Beruf, wie jeder andere auch (...). Es geht hier nicht darum, daß Hausfrauen ein hohes Gehalt verlangen sollen. Es geht darum, daß jeder endlich weiß, was ihre Arbeit in nackten Zahlen wert ist (...) Die Untersuchung beweist zwar, daß eine Hausfrau und Mutter mindestens soviel leistet wie eine Frau im Beruf. Den entscheidenden Unterschied allerdings macht sie nicht deutiich - einen Unterschied, der die Mutter zu Hause eben doch unvergleichbar macht. Sie ist nämlich nicht gleichwertig ersetzbar wie jeder andere Mensch im Beruf. Diesen Wert kann auch der beste Wissenschaftler nicht in Zahlen ausdrücken."

7 Die Zahl umfaßt nur verheiratete Frauen im Alter von 20 und mehr Jahren aus dem Jahre 
monatlich unterstellt. Die pro Monat aufzubringende Lohnsumme würde so 15.4 Milliarden, die Jahressumme 184.8 Milliarden DM betragen (8).

Als Adressaten angesprochen wurden im Verlauf der Arbeit der Ehemann (9) und der „Kapitalist“. Es bleibt zur Vervollständigung der Diskussion der „Staat“ zu ergänzen. Die ökonomische Begründung für die Forderung añ den „Staat ${ }^{66}$ wird an dessen Funktion der Bereitstellung allgemeiner Rahmenbedingungen der Produktion festgemacht.

Eine Lohnforderung an den Ehemann oder den „,Kapitalisten ${ }^{66}$ hätte vermutlich in beiden Fällen eine Lohnerhöhung des Mannes zur Folge. Im Falle des Ehemannes, aufgrund der Tatsache, daß das augenblickliche Durchschnittseinkommen der Mehrzahl der Ehemänner kaum erlauben dürfte, der Ehefrau die unterstellte Summe zur Verfügung zu stellen (10), im Falle des ,Kapitalisten“, aufgrund der Tatsache, daß dieser mit äußerst geringer Wahrscheinlichkeit die Ehefrau zusätzlich zum Mann einstellen würde.

Dies hieße in beiden Fällen, der Frau käme das Geld unmittelbar (Arbeitgeber Mann) oder vermittelt (Arbeitgeber ,Kapitalist") über den Ehemann zu. Ihre Interessenvertretung würde sich entweder direkt gegen den Ehemann richten, oder aber dieser wäre gezwungen, ihre Interessen stellvertretend zu übernehmen.

Der emanzipatorische Charakter einer Lohnforderung unterläge in beiden Fällen erheblichen Einschränkungen.

Das zusätzlich benötigte Geld wäre aufzubringen entweder vom „Kapitalisten“, dies hieße Verteuerung der Produktionskosten mit Auswirkungen auf die Produktionsstruktur, oder Abwälzungen über die Preise, oder aber in Form von Staatszuschüssen, dies hieße Erhöhung des direkten oder indirekten Steueraufkommens. Bliebe der Staat als Adressat einer Lohnforderung.

Die zu zahlende Lohnsumme von $184.8 \mathrm{Mrd}$. DM entspräche nahezu dem gesamten Staatsverbrauch des gleichen Jahres (11).

Die bestehenden Verteilungsstrukturen als gegeben unterstellt, läßt sich konstatieren, daß kein Grund zum Optimismus besteht.

Dennoch kann das Ergebnis nur als logische Konsequenz der Tatsache angesehen werden, daß Hausarbeit bisher nicht bezahlt wurde. Die Forderung als berechtigt anzuerkennen heißt, die Verteilungsstrukturen in Frage zu stellen.

Damit wird die Durchsetzung der Lohnforderung zu einer Machtfrage. Hier erweist sich nicht nur die Einheit der Frauenbewegung vonnöten, d.h. das Eintreten

1975. Nicht eingeschlossen sind gut 1.3 Millionen alleinstehende Mütter. Vgl. Zwischenbericht der Enquete-Kommission..., a.a.O., S. 34

8 Die Jahreslohnsumme entspräche in etwa $20 \%$ des Bruttosozialprodukts 1975

9 Es widerspräche dem Ansatz der "Lohn für die Hausarbeit" Forderung, das Geld vom Ehemann zu fordern. Dieser ist hier nur - in Anlehnung an die Darstellung in Teil 2.4 dieser Arbeit - zur Vervollständigung aller Möglichkeiten herangezogen worden.

10 Das Jahresdurchschnittseinkommen pro Kopf der abhängig Beschäftigten betrug 1975 16 255.- DM (netto). Um die Vorbehalte gegenüber der Aussagekraft von Durchschnittszahlen zu berücksichtigen, sei noch einmal auf den Demonstrationscharakter dieser Rechnung hingewiesen. Quelle: Wirtschaft und Statistik 6/76

11 Der Staatsverbrauch des Jahres 1975 lag bei 221.4 Mrd. DM. Quelle: Statistisches Bundesamt, Sonderauflage der Landeszentrale für politische Bildungsarbeit, Berlin, August 1976 
dafür, daß alle Frauen in und vor allem auch außerhalb des Hauses diese Forderung als eigene begreifen,

es erweist sich weiterhin - bei allem Vorbehalt angesichts der Realität des „Arbeitnehmerpatriarchats“ - die geforderte Einheit der Arbeiterklasse vonnöten, um zu verhindern, daß eine Umverteilung zu ihren Ungunsten verläuft.

Die Lohnforderung - und darin besteht ihre entscheidende Mobilisierungswirkung - wird zu Veränderungen der familialen Organisation zwingen.

Welcher Art diese Veränderungen sein werden, d.h. ob durch Haushaltsverpflichtungen des Mannes die Konfliktlösung weiterhin dem privaten Bereich vorbehalten bleibt, oder, im Falle einer gesellschaftlichen Übernahme der familialen Arbeit, in welcher Form diese stattfinden wird, hängt nicht zuletzt davon ab, inwie weit die geforderte Einheit von Frauen- und Arbeiterbewegung gelingen wird.

Ludmilla Müller, Die Wertlosigkeit der Arbeit der Kinderaufzucht im Kapitalismus, in: PROKLA Nr. 22, 1976

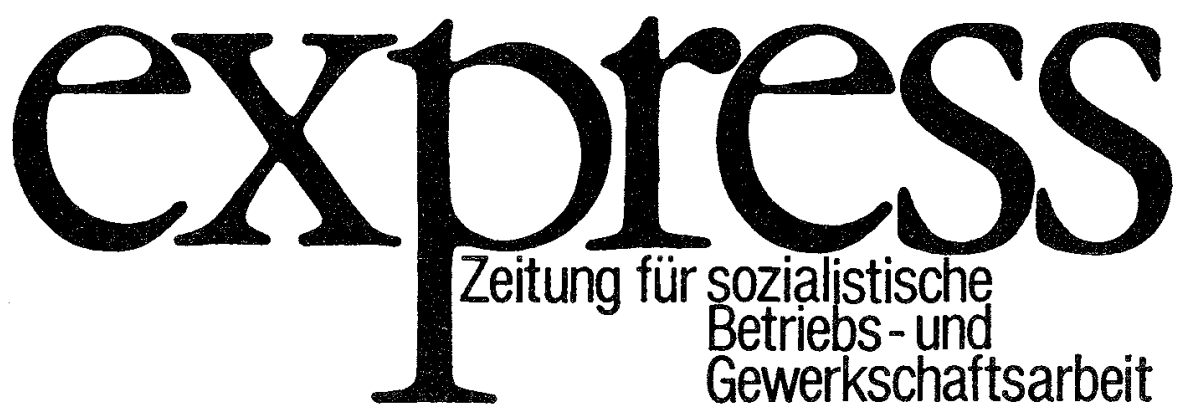

Sprachrohr der Kollegen und Genossen, die sozialistische Betriebs- und Gewerkschaftsarbeit machen. Informationen über die Auseinandersetzung zwischen Kapital und Arbeit. Beiträge, die man nicht in den Gewerkschaftszeitungen findet.

Einzelpreis DM 1,20.

Bezugspreis, jährlich, DM 14,- + DM 6,- Versandkosten

Probeexemplare anfordern bzw. Abonnementsbestellung bei Verlag $2000 \mathrm{GmbH}$, Postfach 591, 605 Offenbach 4. 